\title{
28 Research Square \\ SorCS3 Promotes the Internalization of p75NTR to Inhibit Glioma Progression
}

\section{Yanqiu Zhang}

Harbin Medical University - Daqing Campus

Yue Li

Harbin Medical University - Daqing Campus

\section{Yuhua Fan}

Harbin Medical University - Daqing Campus

\section{Baoshan Zhao}

Harbin Medical University - Daqing Campus

Huan Liang

Harbin Medical University - Daqing Campus

\section{Zhiliang Li}

Harbin Medical University - Daqing Campus

Haiyan Xu

Harbin Medical University - Daqing Campus

Dongliang Li ( $\sim$ lidongliang@hrbmu.edu.cn )

Harbin Medical University

\section{Research Article}

Keywords: SorCS3, p75NTR, Internalization, Glioma

Posted Date: June 8th, 2021

DOI: https://doi.org/10.21203/rs.3.rs-548754/v1

License: (c) (1) This work is licensed under a Creative Commons Attribution 4.0 International License.

Read Full License 


\section{Abstract}

Background: Glioma is a fatal malignancy caused by dysregulation of cellular signal transduction. Internalization plays a key role in maintaining signalling balance. SorCS3 is involved in nerve cell receptor internalization. However, the impact of SorCS3 on the biological processes involved in glioma has not yet been reported. Here, we highlight the potential of SorCS3-mediated regulation of signalling receptor internalization as a rational target for therapeutic intervention in glioma.

Methods: SorCS3 expression was analysed in the TCGA and CGGA databases and in tissue microarrays. The effects of SorCS3 on the proliferation and metastasis of glioma cells were examined in vitro and in vivo with Transwell, wound healing, EdU incorporation and nude mouse tumorigenicity assays.

Fluorescent 5-FAM, SE-labelled proteins were used to detect the internalization of SorCS3 in glioma cells. Immunofluorescence and Co-IP assays were conducted to investigate the downstream effector of SorCS3. Moreover, Dynasore and Ro 08-2750, inhibitors of internalization and NGF binding to p75NTR, respectively, were used to validate the biological functions of SorCS3 in glioma.

Results: Our data demonstrated that SorCS3 was downregulated in glioma tissues and closely related to favourable prognosis. Overexpression of SorCS3 inhibited the proliferation and metastasis of glioma cells in vitro and in vivo, while silencing of SorCS3 exerted the opposite effects. Mechanistic investigations showed that SorCS3 bound to P75NTR, which subsequently increased the internalization of p75NTR, and then transported p75NTR to the lysosome for degradation, ultimately contributing to inhibition of glioma progression.

Conclusions: Our work suggests that SorSC3 is a marker of promising prognosis in glioma patients and suggests that SorCS3 regulates internalization, which plays an important role in inhibiting glioma progression.

\section{Background}

Glioma is the most common type of primary intracranial tumour, with high mortality and recurrence rates and a low cure rate; it has a 5-year relative survival rate of only $5-8 \%$ [1]. Currently, the incidence of glioma is increasing annually. Due to the specific location and high metastasis rate of glioma and the complexity of its molecular mechanism of occurrence and development, the prevention and treatment of glioma remains a global challenge, although it has been studied for decades. Hence, the study of glioma pathogenesis and discovery of key glioma biomarkers could facilitate the diagnosis and treatment of glioma recurrence.

Sortilin related VPS10 domain containing receptor 3 (SorCS3) belongs to the vacuolar protein sorting 10 protein (VPS10p) receptor family. VPS10p domain receptors are a unique class of sorting receptors that direct the intracellular transport of target proteins between the cell surface, endosomes, Golgi and lysosomes in mammalian cells, and these proteins have receptor internalization abilities [2]. One of the characteristic functions of VPS10p family members is the regulation of ligand-induced internalization of 
receptors [3]. Analysis of the SorCS3 domain revealed that the short intracellular C-terminus contains consensus signals for rapid internalization [4]. We speculated that SorCS3 may have an internalization function similar to that of other VPS10p family members. In addition, receptor internalization plays an important role in regulating receptor signal transduction; thus, affecting internalization has the potential to influence cellular function [5].

Earlier studies focused on roles of SorCS3 in the control of protein transport in neurons and disorders of systemic metabolism [6]. SorCS3 is a specific receptor for nerve growth factor (NGF) that regulates NGF signal transduction across membranes, and SorCS3 is mainly expressed in the hippocampus and cortex of the brain. However, the clinical relevance and physiological significance of the interaction between SorCS3 and NGF have not yet been fully elucidated. Thus, the exact role of SorCS3 in glioma remains unclear.

Abnormal activation of signal transduction pathways, aberrant regulation of cell proliferation, and ectopic metastasis are key events in glioma development. Generally, dysregulated expression of growth factors and their receptors is the core component underlying abnormal activation of signal transduction pathways. NGF and NGF receptors are important in the progression of some neurological diseases, including tumours [7-9]. NGF regulates cell behaviour by binding to two different receptors, TrkA and p75NTR. In addition, it has been confirmed that p75NTR is an important and potent mediator of invasion in human glioma. However, it is currently unclear whether the p75NTR signal is propagated via internalization regulation or via other mechanisms.

In the present study, we revealed a novel molecular mechanism by which SorCS3 promotes internalization to regulate glioma progression. Our results revealed that SorCS3 interacts with p75NTR but not TrkA. SorCS3 regulates $\mathrm{p} 75$ NTR by controlling its internalization from the plasma membrane to the lysosome, thereby limiting signal transduction, an essential driving force of tumour aggressiveness. Moreover, we found that low expression of SorCS3 is associated with a high degree of glioma malignancy. Hence, SorCS3 expression is a marker of favourable prognosis in glioma patients. Taken together, our results reveal a novel mechanism of regulated receptor signal transduction and suggest a promising receptor signal blocker for antitumour therapy.

\section{Materials And Methods}

\section{Cell lines and cell culture}

The glioblastoma cell lines U-87 MG (U87) and U251 were purchased from the Type Culture Collection of the Chinese Academy of Sciences, Shanghai, China. The cell lines LN229 and T98 were obtained from the First Affiliated Hospital of Harbin Medical University. These cells were grown in Dulbecco's modified Eagle's medium (HyClone, SH3002201) supplemented with $10 \%$ foetal bovine serum (Biological Industries, 04-001-1A) and 1\% (vol/vol) penicillin/streptomycin (Beyotime, C0222) in an incubator $\left(37^{\circ} \mathrm{C}\right.$, $\left.5 \% \mathrm{CO}_{2}\right)$. In the follow-up experiment, cells at passage 5 to 15 that exhibited good proliferation were 
selected. According to the specifications of the cell culture flask or culture plate, appropriate complete medium was added.

\section{TCGA and CGGA database analysis}

By analysing publicly available datasets from The Cancer Genome Atlas (TCGA) and the Chinese Glioma Genome Atlas (CGGA), we examined the levels of SorCS3 and p75NTR expression in human glioma samples. SorCS3 and p75NTR levels were significantly changed in glioma samples compared with control samples.

\section{Transient transfection}

In this research, we carried out transfection for overexpression and knockdown with cells in serum-free and antibiotic-free medium and at a confluence of $80-90 \%$. We used Lipofectamine 3000 reagent for overexpression plasmid transfection following the supplier's instructions. A Flag tag was fused to the carboxyl end of the SorCS3 gene open reading frame (ORF) to construct the SorCS3 overexpression plasmid in the pCMV3-C-Flag vector. The vector was purchased from Sino Biological. Another pCMV3-CFlag vector without the ORF region of SorCS3 was used as a negative control. X-treme GENE siRNA Transfection Reagent (Roche) was used to complete transient transfection of small interfering RNAs (siRNAs), which were purchased from Sangon Biotech (Shanghai, China). si-SorCS3-1\# and si-SorCS3-2\# were applied for SorCS3 gene knockdown. si-GAPDH was used as the positive control. The internalization inhibitor Dynasore (GIpBio) was added to transfected cells. The binding of Ro 08-2750 (MCE) to NGF inhibits the interaction of NGF and p75NTR, and Ro was added to cells transfected with the pCMV3SorCS3-Flag plasmid.

\section{Wound healing assay}

Before cell seeding, we marked the bottom of a 6-well plate to facilitate subsequent imaging and positioning. After 4 hours of transfection, the medium containing the transfection reagent was discarded. After 24 hours, a $200 \mu \mathrm{L}$ pipette tip was used to scratch an even wound on the adherent cells in the culture plate, and the cells were washed with phosphate-buffered saline (PBS) to replace the medium with low-serum medium. Images were acquired with a microscope, and the wound width was recorded at 0 hours, 12 hours, and 24 hours, until 48 hours after wound closure. Image J was used to analyse the distance of wound closure. The migration distance at different points in the treatment group was determined, and the migration distance in the control group was used as a reference. After normalization and statistical analysis, the wound closure (migration) in the experimental group compared to the control group was calculated as a percentage.

\section{Colony formation assay}

Cells ( 2000 cells/well) were plated in 6 -well plates. After 7 days, colonies ( $>50$ cells) were counted under a microscope, fixed with $75 \%$ ethanol and stained with crystal violet staining solution. The colonies were imaged and analysed. 


\section{Transwell migration and invasion assays}

The membrane in the upper chamber of the Transwell apparatus was coated with precooled Matrigel to evaluate the invasion ability. Matrigel was diluted in serum-free medium at a volume ratio of 1:8. Twentyfour hours after transfection, cells $\left(2 \times 10^{4}\right.$ cells in a final volume of $200 \mu \mathrm{L}$ of low-serum medium) were seeded in the upper chamber. Then, $500 \mu \mathrm{l}$ of complete medium was added to the lower chamber, and both chambers were incubated for 24 hours. The upper chamber was transferred to $75 \%$ alcohol for fixation for more than 30 minutes and stained in crystal violet solution $(0.5 \%)$ for more than 30 minutes. Residual crystal violet solution was removed by washing with water. Invaded cells were imaged under a microscope. The addition of Matrigel was not required for evaluating the migration ability of glioma cells. The subsequent steps were the same as those described above for evaluation of the invasion ability.

\section{Western blot analysis}

Total protein was extracted using RIPA buffer, and protein expression was analysed by western blotting as described previously [10]. $\beta$-Actin was used as the endogenous control. Information about the antibodies utilized for western blotting is listed in Additional file 4: Table S1. Images were acquired using a UVP ChemStudio-PLUS imaging system, and the density of bands was measured with ImageJ software.

\section{Quantitative real-time polymerase chain reaction}

Total RNA was isolated with TRIzol Reagent (Invitrogen) according to the manufacturer's protocol. The concentration of total RNA was detected with a NanoDrop One spectrophotometer (Thermo Scientific). Reverse transcription was performed using a PrimeScript $\otimes 1$ st Strand cDNA Synthesis Kit (Takara) and $2000 \mathrm{ng}$ of total RNA according to the manufacturer's protocol. Rotor-Gene Q Series Software (QIAGEN) was used to conduct qPCR. Gene expression of SorCS3 and GAPDH in glioma cells was analysed using custom-designed primers and SYBR Green Real-time PCR Master Mix (TOYOBO). The comparative Ct method was used for relative quantification of target mRNA levels, with GAPDH as the negative control.

\section{5-FAM, SE-BSA internalization assay}

5-FAM, SE (Invitrogen) is a fluorescent labelling reagent that exists as a single isomer to bind peptides, proteins and nucleotides via chemical reactions. According to the manufacturer's protocol, fluorescently labelled bovine serum albumin (BSA) was prepared by incubation in the dark. 5-FAM, SE-BSA collected from the cut-off column was filtered through a PVDF filter to remove bacteria. 5-FAM, SE-BSA was added to cultured glioma cells to observe the internalization ability under a fluorescence microscope.

\section{Co-Immunoprecipitation}

Co-immunoprecipitation (Co-IP) was performed using a Pierce Co-IP Kit (Thermo Scientific) according to the manufacturer's protocol. To study protein:protein interactions, U251 cells in monolayer culture were lysed for detection of interactions. Isolation of native protein complexes from lysates of U251 cells was 
performed. According to experimental needs, a purified anti-Flag antibody or anti-p75NTR antibody was used to immunoprecipitate the antigen, and any co-immunoprecipitated interacting proteins were immobilized directly onto an amine-reactive resin by covalent coupling. The final protein eluate was denatured and analysed by western blotting.

\section{Immunohistochemistry}

Immunohistochemistry (IHC) was performed on tissue microarrays by monoclonal antibodies against SorCS3 and p75NTR under the appropriate conditions. Following routine immunohistochemical procedures, the expression of genes in the tissue microarrays was evaluated by microscopy. An ethical certificate issued by the hospital from which the clinical samples was obtained was acquired for use of the tissue microarrays.

\section{Cell proliferation assays}

An EdU incorporation assay was used to assess cell proliferation according to the manufacturer's protocol (RiboBio). After transfection, $2.0 \times 10^{3}$ cells were seeded into each well of a Lab-Tek ${ }^{\text {TM }}$ II Chamber Slide (Thermo Scientific). EdU was incorporated into proliferating cells during a 2-hour incubation. EdU incorporation was detected with a fluorescently labelled azide through a catalysed reaction by fluorescence microscopy.

\section{Immunofluorescence staining and imaging}

Immunofluorescence staining was performed using Lab-Tek ${ }^{\mathrm{TM}}$ II Chamber Slides (Thermo Scientific). Cells $\left(2.0 \times 10^{3}\right)$ were seeded into each well. The medium in the wells was discarded before cells were postfixed with 4\% PFA for 1 hour at room temperature. Adhered cells were rinsed in PBS three times and permeabilized with $0.5 \%$ Triton X-100 for 1 hour at room temperature. After blocking for 1 hour in $5 \%$ BSA, cells in each well were incubated with the primary antibody against the target protein in a black wet box at $4{ }^{\circ} \mathrm{C}$ overnight. Cells were then incubated with the corresponding secondary antibody at room temperature in the dark. DAPI was added to the wells at room temperature for 30 minutes. The chamber slide was carefully removed from the original packaging and observed via fluorescence microscopy.

\section{Nude mouse tumorigenicity assay}

Fourteen male nude mice were used in the tumorigenicity assays. Our study was approved by the Ethics Committee of Harbin Medical University (Daqing). Nude mice were randomly grouped into the control group and the OE-SorCS3 group. Mice were inoculated subcutaneously in the right flanks with $1 \times 10^{7}$ U87 cells transfected with the negative control vector or the pCMV3-SorCS3-Flag plasmid.

\section{Statistical analysis}

SPSS was used for statistical analysis. All values are expressed as the means \pm SEMs, and all experiments were repeated at least three times. Student's $t$-test was used to determine the statistical 
significance of the differences between groups. A comparative t-test was used for analysis of clinical samples. Statistical comparisons among multiple groups were carried out using analysis of variance (ANOVA) followed by Dunnett's test. Differences with $P<0.05$ were considered significant $\left({ }^{\star} P<0.05,{ }^{\star \star} P\right.$ $<0.01, * \star \star P<0.001)$.

\section{Results}

Low levels of SorCS3 expression in human glioma cancer tissues are associated with poor clinical outcomes.

To explore the potential roles of SorCS3 in glioma, we analysed RNA sequencing results from The Cancer Genome Atlas (TCGA) database and found that the mRNA level of SorCS3 was decreased in glioma (Fig. 1A). Further, we found that the mRNA level of SorCS3 in high-grade glioma was much lower than that in low-grade glioma (Fig. 1B). Using two independent datasets, we demonstrated that a low level of SorCS3 transcripts in low-grade glioma and high-grade glioma tissues was associated with shorter overall survival times in patients (Fig. 1C and 1D). In contrast to low expression, high expression of SorCS3 was significantly correlated with better survival. Taken together, these data demonstrated that SorCS3 is significantly downregulated in glioma, suggesting that it may function as a tumour suppressor.

SorCS3 inhibits glioma cell migration, invasion and proliferation in vitro.

To investigate the biological effects of SorCS3 in glioma progression, and considering that SorCS3 has not previously been studied in glioma cells, we first examined cellular SorCS3 levels in a quantitative manner. As shown in Fig. 2A, western blot analysis showed that SorCS3 expression was significantly decreased in the U251 cell line compared to the other glioma cell lines tested (U87, A172, and T98). Next, we performed cell proliferation, invasion and migration, apoptosis, and cell cycle assays in two glioma cell lines (U87 and U251) after transfection with the pCMV3-SorCS3-Flag plasmid (Fig. 2B). Further, we examined the effect of SorCS3 on the invasion and migration abilities of glioma cells. In vitro wound healing and Transwell assays demonstrated that overexpression of SorCS3 reduced the migratory and invasive potential of tumour cells (Fig. 2C-F). In addition, SorCS3 overexpression reduced the proliferation of glioma cells, as determined by an EdU incorporation assay (Fig. $2 \mathrm{G}$ ) and a plate colony formation assay (Fig. 2H). Given that SorCS3 regulates cell proliferation, we examined whether SorCS3 regulates cell cycle progression or cellular apoptosis. We found that SorCS3 expression in U251 cells decreased the number of cells in S phase but increased the number of cells in G0/G1 phase (Supplementary Fig. S1A). However, overexpression of SorCS3 had no effect on apoptosis (Supplementary Fig. S1B). These findings showed that SorCS3 significantly attenuates the growth of glioma cells in vitro.

Hence, we examined the effects of SorCS3 on the expression of EMT and proliferation markers. Western blot analysis showed that in SorCS3-overexpressing glioma cells, the protein expression of the epithelial marker E-cadherin was upregulated, while the expression of the mesenchymal marker vimentin as well as the transcriptional repressors Snail and PCNA was markedly decreased (Fig. 2I). These results suggested 
that SorCS3 suppresses the proliferation and invasion of glioma cancer cells, functioning as an inhibitor of glioma development.

To verify the tumour suppressor roles of SorCS3, we first silenced SorCS3 expression using two siRNA constructs (si-SorCS3-1\# and 2\#) in U251 and U87 cells (Fig. 3A-B). Knockdown (KD) of SorCS3 significantly increased the invasion and migration of glioma cells compared to that of control cells in the Transwell assay (Fig. 3C-D and Supplementary Fig. S2A-B). Accordingly, we observed similar patterns in the wound healing assay (Fig. 3E-F and Supplementary Fig. S2C-D). Similarly, we found that knockdown of SorCS3 significantly enhanced the proliferation of glioma cells (Fig. 3G-H and Supplementary Fig. $\mathrm{S} 2 \mathrm{E}$ ). Upregulation of SorCS3 resulted in a reduction in glioma cell proliferation, which was blocked by siSorCS3. In contrast, silencing SorCS3 resulted in obvious promotion of cell proliferation. Further, western blot analysis showed that in SorCS3-KD glioma cells, the protein levels of the EMT markers E-cadherin and Vimentin were significantly changed, while those of the transcriptional repressors Snail and PCNA were markedly increased (Fig. 3I). In summary, we concluded that SorCS3 functions as a tumour suppressor gene in glioma cells in vitro.

SorCS3 and p75NTR have a negative regulatory relationship.

The VPS10p domain receptor SorCS3 has been implicated in several pathways of protein internalization and sorting between the plasma membrane and endosomes. Although findings from an earlier report indicate that SorCS3 binds NGF and that NGF is the driving force of glioma cell progression [9], the functional implications of these interactions are poorly understood [11]. In addition, NGFR (TrkA and p75NTR) has been verified to play an important role in glioma progression. We speculated that SorCS3 is involved in the impact of increased tumour-promoting behaviour upon NGF-induced NGFR internalization. First, we explored the specific ligand-induced internalization ability of SorCS3 in glioma cell lines. Fluorescence microscopy revealed that the overexpression of SorCS3 on the cell membrane surface increased the internalization of albumin. Moreover, silencing SorCS3 suppressed the specific ligandinduced internalization ability and decreased the appearance of bright fluorescent puncta on the cell membrane surface (Fig. 4A-B). Next, we validated the correlation between the SorCS3 and NGFR levels after transfection with the SorCS3 overexpression plasmid or SorCS3 siRNA. Our findings showed that the SorCS3 and p75NTR levels were negatively related but that the SorCS3 and TrkA levels were not significantly related (Fig. 4C). Therefore, we analysed the correlation between the mRNA levels of SorCS3 and p75NTR. Interestingly, the results showed a weak negative correlation between these levels (Fig. 4D). Therefore, there might be another stronger regulatory relationship between SorCS3 and p75NTR. Previous studies have shown that p75NTR dramatically enhances the migration and invasion of genetically distinct glioma cells and frequently exhibits robust expression in specimens from patients with highly invasive glioblastoma [12]. We verified the biological significance of p75NTR in glioma. Kaplan-Meier analysis indicated that high expression of p75NTR was negatively correlated with the overall survival time of glioma patients (Fig. 4E). Further, we examined the expression of p75NTR in glioma samples of different grades by tissue microarray analysis (Fig. 4F). Taken together, these data indicated that SorCS3 regulates the expression of p75NTR in glioma. 
SorCS3 interacts with p75NTR on the plasma membrane.

Intrigued by the co-internalization of SorCS3 and p75NTR, we generated a SorCS3-Flag fusion protein and then performed a set of Co-IP assays to investigate whether SorCS3 and p75NTR interact. First, we used an anti-Flag antibody to pull down p75NTR, and as shown in Fig. 4G, SorCS3 and p75NTR may interact. However, there seemed to be no interaction between SorCS3 and TrkA. Second, we found that endogenous p75NTR and SorCS3 co-precipitated in U87 cells, indicating that SorCS3 and p75NTR may exist in the same protein complex (Fig. 4H). Next, we sought to examine the subcellular localization of endogenous p75NTR and SorCS3 in U87 cells with high levels of SorCS3 expression. In U87 cells, endogenous SorCS3 colocalized with p75NTR (Fig. 4I). In addition, a portion of SorCS3 was demonstrated to have a distict overlap with EEA1 and Rab5, indicating its localization in early endosomes. Previous studies have indicated that p75NTR is mainly confined to the plasma membrane and overlaps with endosomes during internalization and sorting [13]. We speculated that p75NTR may be involved in the internalization process regulated by SorCS3.

Taken together, these findings indicated that SorCS3 co-precipitates with p75NTR. However, at this point, we do not know whether SorCS3 interacts directly with p75NTR or whether they are coupled through a molecular complex or an intermediary partner.

NGF stimulation promotes the interaction between SorCS3 and p75NTR.

We then sought to investigate the mechanism underlying the SorCS3-p75NTR interaction. We stimulated U87 human glioma cells with NGF under normal serum conditions, investigated whether NGF stimulation promoted the interaction between p75NTR and SorCS3, and analysed the canonical internalization pathways of SorCS3 in whole-cell lysates (WCLs). In addition, we explored whether the interaction between p75NTR and SorCS3 increases with NGF treatment (Fig. 5A). We speculated that NGF may act as a bridge or linker between P75NTR and SorCS3, forming a complex with these proteins to complete the internalization process. Subsequently, we measured the expression level of p75NTR in SorCS3overexpressing glioma cells at different time points after NGF treatment. Unexpectedly, in cells with SorCS3 overexpression, the expression of p75NTR gradually decreased with increasing NGF treatment time. Interestingly, the expression level of the lysosomal marker Lamp2 was increased by stress, but the expression of the late endosome marker Rab7 was not changed (Fig. 5B). These results showed that the decreased level of p75NTR may result from its degradation through the lysosomal pathway. As NGF treatment triggered decreased intracellular retention of p75NTR in cells with SorCS3 overexpression, we investigated the subcellular localization of SorCS3 in U87 cells. Under NGF treatment conditions, immunofluorescence experiments showed that the overlap of SorCS3 with p75NTR and RAB5 was increased (Fig. 5C). Subsequently, we investigated whether overexpression of SorCS3 can impair the canonical p75NTR signalling pathway in cells treated with NGF. Overexpression of SorCS3 impaired p75NTR signalling, as evidenced by the decreased phosphorylation of both AKT and ERK1/2. After treatment with NGF, the inhibitory effect on the p75NTR signal was enhanced to some extent. In addition, SorCS3-depleted cells exhibited sustained p75NTR signalling (Fig. 5D). 
Previous studies have shown that NGF is required for glioma cell invasion and proliferation [8]. To verify the role of NGF in SorCS3-mediated inhibition of glioma, we analysed the biological functions of metastasis and proliferation in SorCS3-overexpressing glioma cells treated with NGF (Fig. 6A-B). Under normal conditions, NGF treatment promoted the invasion and metastasis of glioma cells. However, when SorCS3 was overexpressed, the effect of NGF was blocked in two glioma cell lines (U87 and U251). Taken together, these results suggested that SorCS3 can weaken the cancer-promoting effect of NGF and that NGF can enhance the SorCS3-regulated internalization of p75NTR.

Inhibition of internalization abolishes the tumour-suppressive ability of SorCS3.

We then sought to investigate the underlying mechanism by which SorCS3 inhibits glioma. We inhibited endocytosis using Dynasore, a cell internalization inhibitor. To prevent the inhibition of Dynasore internalization, we also added NGF to promote internalization mediated by SorCS3. In Dynasore-treated cells, the inhibitory effect of SorCS3 on migration and invasion was abolished (Fig. 7A). As expected, in the EdU incorporation assay, the number of proliferating cells did not change significantly (Fig. 7B). Subsequently, we investigated whether suppression of internalization impairs the SorCS3-p75NTR signalling pathway. Interestingly, in cells with SorCS3 overexpression, the decrease in the p75NTR level was alleviated by the inhibition of internalization. In addition, blockade of internalization suppressed the change in p75NTR signalling, as evidenced by the unchanged phosphorylation of both AKT and ERK1/2 (Fig. 7C). To further verify that the biological function of SorCS3 in suppressing cancer is achieved through the NGF/p75NTR signalling pathway, we selected the competitive NGF inhibitor Ro 08-2750 to inhibit binding between endogenous NGF and p75NTR. As a result, we found that Ro 08-2750 restored the influence of SorCS3 on the downstream p75NTR signalling pathway. Taken together, these findings indicated that inhibiting the internalization of or the binding between NGF and SorCS3 interfered with the tumour suppressor function of SorCS3.

SorCS3 downregulation is associated with poorer prognosis.

Our results obtained from animal models revealed that overexpression of SorCS3 is associated with inhibited cellular proliferation and retarded tumour growth (Fig. 8A). To determine whether this phenomenon is also relevant to human glioma, we performed immunohistochemical analysis on glioma tissue microarrays containing samples from 108 patients. We analysed SorCS3 expression according to grade, with grades I-IV corresponding to well-differentiated to moderately differentiated tumours. Low levels of SorCS3 protein were found to positively correlate with greater tumour differentiation in the tissue microarray analysis (Fig. 8B). These results demonstrate that SorCS3 acts as a novel tumour suppressor and is negatively correlated with the degree of glioma malignancy.

\section{Discussion}

SorCS3 is a type I transmembrane protein that mediates the signalling and/or intermembrane transport of ligands and is regarded as a sorting receptor with important neuronal functions. Previous studies have shown that SorCS3 is exclusively expressed in the brain with tissue specificity. However, we detected the 
expression of SorCS3 in glioma tissue microarray samples and cell lines (Fig. 1E-F and Fig. 7A). In addition, we analysed data from the TCGA database and found that the expression of SorCS3 in gliomas is significantly reduced. The molecular characteristics of glioma classification are crucial for the treatment and prognosis of glioma. In the Chinese Glioma Genome Atlas (CGGA) dataset, we evaluated the correlations between SorCS3 expression and histological type, clinical factors, and molecular characteristics. Low expression of SorCS3 was closely related to IDH wild-type gliomas, 1p/19q gliomas, and recurrent gliomas (Fig. S3A-F). However, there was no association with sex (Fig. S3G). These findings indicate that SorCS3 may have important biological functions in gliomas.

What triggers the biological function of SorCS3 as a tumour suppressor in glioma cells? The oncogenic and tumour-suppressive functions of molecules likely depend on the mechanism involved [14]. According to the biological characteristics of SorCS3, which may be involved in the process of metastasis and proliferation signalling pathways, SorCS3 acts as a regulator of cell membrane receptor internalization.

Abnormal signal transduction is one of the main characteristics of tumour progression. It is the result of cooperation among multiple driving factors. In addition to gene mutation, epigenetic modification, and transcriptome regulation, internalization directly leads to receptor degradation or recycling to regulate the activation of signalling pathways. For example, Fabrice et al. found that Sortilin is a regulator of EGFR intracellular trafficking that promotes receptor internalization and limits signalling, which in turn impacts tumour growth [15]. Some studies have shown that SORLA, through interactions at its extracellular domain, exists in a complex with HER2 and co-traffics with HER2, facilitating HER2 recycling to the plasma membrane to support HER2 downstream signalling. In the absence of SORLA, HER2 becomes localized to enlarged, partially dysfunctional lysosomes, resulting in defective HER2 signalling and increased sensitivity $[16,17]$. Increasing evidence indicating the important roles that Sortilin and SorLA participate in a variety of tumour progression-related processes. SorCS3, Sortilin and SorLA are members of the same VPS10p family; they have similar biological functions and are highly conserved among different species. We speculate that SorCS3-mediated cellular internalization is one of the key mechanisms for suppressing glioma metastasis and proliferation. Our data also confirmed this suppression (Fig. 4A).

NGF, by binding to two specific membrane receptors, TrkA and p75NTR, activates different downstream signalling cascades [18]. SorCS3 is an NGF receptor and can bind to NGF [11]. However, the relationship between SorCS3 and the other two NGFRs has not been investigated. It is not known whether these receptors act independently in competitively binding to NGF or bind to each other to form a complex. In addition, the relationship between SorCS3 and p75NTR is not yet clear. We attempted to verify the interaction between SorCS3 and p75NTR using co-immunoprecipitation assays and found that this interaction might exist (Fig. 4H). In glioma, p75NTR has been reported to be an important regulator of cell viability, invasion, and migration $[12,19,20]$. For example, p75NTR regulates invasion and progression through $\gamma$-secretase-dependent and $\gamma$-secretase-independent mechanisms in glioma [21, 22]. Further, phosphorylation of p75NTR on S303 and S425 is necessary for glial tumour invasion [23]. 
p75NTR can delay the internalization and degradation of TrkA after NGF treatment to promote the potentiation of TrkA signalling [24]. However, the mechanism by which NGF regulates the internalization of p75NTR is still unclear. Our studies showed that SorCS3 interacts with p75NTR. By immunofluorescence, p75NTR was found to be internalized via cell membrane receptor-mediated internalization. However, at this point, we do not know whether SorCS3 interacts directly with p75NTR or whether they are coupled through a molecular complex or an intermediary partner. We speculate that NGF may act as a bridge that links the SorCS3 and p75NTR proteins, since the SorCS3-p75NTR interaction was markedly increased by NGF treatment. In addition, other research has shown that proNGF can form a ternary complex with Sortilin or SorCS2 and p75NTR as co-receptors, which do not interact directly but are separated by the proNGF dimer, which acts as a separating wedge $[25,26]$. This is very similar to the predicted SorCS3-p75NTR interaction model.

In our study, NGF treatment accelerated the routing of p75NTR towards rapid internalization and degradation by SorCS3 (Fig. 5B). However, it is still unclear whether p75NTR degradation is controlled solely by the lysosomal pathway or whether other mechanisms, such as autophagy, are also involved. Many classic studies have shown that once internalized from the plasma membrane, membrane-bound vesicles that carry receptors (VPS10p family members) from the cell surface fuse with the early endosome. The early endosome (EE) serves as a sorting station from which cargoes are either delivered to the endolysosomal system for degradation or are recycled directly or indirectly to the plasma membrane via the endocytic recycling compartment; endocytic recycling is primarily a default pathway whereby proteins, in the absence of selective signals to target them for degradation, are typically recycled [27]. For example, VPS10p family members can transport many interacting proteins to the plasma membrane via the endocytic pathway and the lysosome for degradation [28-30]. In addition, sphingolipid activator proteins, acid sphingomyelinase, and cathepsin $\mathrm{D}$ and $\mathrm{H}$ have been shown to be trafficked by Sortilin to the lysosome [31, 32]. Receptor internalization and subsequent trafficking to the lysosome promotes protein degradation and is a key biological process in terminating signal transduction [33]. In our study, after SorCS3 overexpression, Rab7 association with the internalization marker showed no change, while Rab7 association with the lysosome marker LAMP2 increased with NGF treatment time. This finding also suggests that SorCS3 can function as a tumour suppressor by regulating signalling receptor internalization and transporting receptors to the lysosome for degradation.

However, this study has several limitations. First, blocking internalization with Dynasore did not completely abolish SorCS3-induced p75NTR degradation (Fig. 7C). This failure is possibly due to other alternative degradation pathways involved in the effects of SorCS3, including autophagy, ubiquitination and proteasomal degradation pathways. Second, we were unable to demonstrate that SorCS3 expression is a reliable prognostic factor for the recurrence and metastasis of glioma. This shortcoming may reflect the inadequate sample size and the incompleteness of patient characteristics, given that information from our study made it impossible to identify SorCS3 as a reliable prognostic factor.

Although extensive studies have been performed to investigate the mechanism of SorCS3 in neurologicalassociated pathways, the SorCS3-mediated mechanism underlying glioma pathogenesis remains 
unclear. The findings of this study may facilitate to the development of novel therapeutic strategies for glioma.

\section{Conclusions}

In this study, we demonstrate that SorCS3, a sorting protein previously not investigated in carcinomas, is expressed at low levels in glioma cell lines and patients. In this model, SorCS3 regulates rapid internalization of p75NTR by forming a complex with this receptor and coupling it to lysosomal degradation, limiting migration and proliferation signalling via p75NTR (Fig. 8C).

\section{Abbreviations}

\begin{tabular}{|ll|}
\hline SorCS3 & Sortilin Related VPS10 Domain Containing Receptor 3 \\
\hline NGF & Nerve Growth Factor \\
\hline ORF & Open Reading Frame \\
\hline EE & Early Endosome \\
\hline IHC & Immunohistochemistry Assays \\
\hline Co-IP & Co-Immunoprecipitation \\
\hline GBM & Glioblastoma \\
\hline IDH & Isocitrate Dehydrogenase \\
\hline LGG & Lower-Grade Glioma \\
\hline TCGA & The Cancer Genome Atlas \\
\hline CGGA & Chinese Glioma Genome Atlas \\
\hline
\end{tabular}

\section{Declarations}

\section{- Ethics approval and consent to participate}

The experimental protocol for animal studies was reviewed and approved by the Harbin Medical

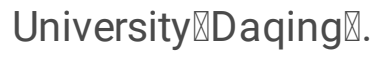

\section{- Consent for publication}

All authors agreed on the manuscript.

- Availability of data and materials 
All data generated or analysed during this study are included within the article or available from the corresponding author on reasonable request.

\section{- Competing interests}

The authors have declared that no competing interest exists.

\section{- Funding}

This study was supported by the Scientific Research Project of Heilongjiang Provincial Health Commission (2019-074), the Heilongjiang Province Postdoctoral Science Foundation (LBH-Z18208) and the China Postdoctoral Science Foundation (2019M661297).

\section{- Authors' contributions}

$D L, Y Z$ and $H X$ conceived the project. DL, $Y Z$ and $Y L$ designed most experiments. $D L, Y L$ and $Z L$ performed the in vitro function and molecular mechanism experiments and analyzed the data. $\mathrm{YF}$ performed the in vivo experiments. BZ for help with the tissue microarrays IHC analysis. All authors read and approved the final manuscript.

\section{- Acknowledgements}

We are grateful to Xiuli Wang for technical help.

\section{References}

1. Ostrom QT, Patil N, Cioffi G, Waite K, Kruchko C, Barnholtz-Sloan JS. CBTRUS Statistical Report: Primary Brain and Other Central Nervous System Tumors Diagnosed in the United States in 20132017. Neuro Oncol. 2020; 22: iv1-iv96.

2. Subkhangulova A, Malik AR, Hermey G, Popp O, Dittmar G, Rathjen T, et al. SORCS1 and SORCS3 control energy balance and orexigenic peptide production. EMBO Rep. 2018; 19.

3. Reitz C. The role of intracellular trafficking and the VPS10d receptors in Alzheimer's disease. Future Neurol. 2012; 7: 423-31.

4. Hermey G, Riedel IB, Hampe W, Schaller HC, Hermans-Borgmeyer I. Identification and characterization of SorCS, a third member of a novel receptor family. Biochem Biophys Res Commun. 1999; 266: 34751.

5. Khailaie S, Rowshanravan B, Robert PA, Waters E, Halliday N, Badillo Herrera JD, et al. Characterization of CTLA4 Trafficking and Implications for Its Function. Biophys J. 2018; 115: 133043.

6. Hermey G, Hoffmeister-Ullerich SA, Merz B, Gross D, Kuhl D, Kins S. Amyloidosis causes downregulation of SorLA, SorCS1 and SorCS3 expression in mice. Biol Chem. 2019; 400: 1181-9. 
7. Walsh EM, Kim R, Del Valle L, Weaver M, Sheffield J, Lazarovici P, et al. Importance of interaction between nerve growth factor and alpha9beta1 integrin in glial tumor angiogenesis. Neuro Oncol. 2012; 14: 890-901.

8. Lawn S, Krishna N, Pisklakova A, Qu X, Fenstermacher DA, Fournier M, et al. Neurotrophin signaling via TrkB and TrkC receptors promotes the growth of brain tumor-initiating cells. J Biol Chem. 2015; 290: 3814-24.

9. Cicvaric A, Yang J, Krieger S, Khan D, Kim EJ, Dominguez-Rodriguez M, et al. The brain-tumor related protein podoplanin regulates synaptic plasticity and hippocampus-dependent learning and memory. Ann Med. 2016; 48: 652-68.

10. Li D, Zhang Y, Zhang H, Zhan C, Li X, Ba T, et al. CADM2, as a new target of miR-10b, promotes tumor metastasis through FAK/AKT pathway in hepatocellular carcinoma. J Exp Clin Cancer Res. 2018; 37: 46.

11. Oetjen S, Mahlke C, Hermans-Borgmeyer I, Hermey G. Spatiotemporal expression analysis of the growth factor receptor SorCS3. J Comp Neurol. 2014; 522: 3386-402.

12. Johnston AL, Lun X, Rahn JJ, Liacini A, Wang L, Hamilton MG, et al. The p75 neurotrophin receptor is a central regulator of glioma invasion. PLoS Biol. 2007; 5: e212.

13. Chao MV. Neurotrophins and their receptors: a convergence point for many signalling pathways. Nat Rev Neurosci. 2003; 4: 299-309.

14. Marisetty AL, Lu L, Veo BL, Liu B, Coarfa C, Kamal MM, et al. REST-DRD2 mechanism impacts glioblastoma stem cell-mediated tumorigenesis. Neuro Oncol. 2019; 21: 775-85.

15. Al-Akhrass $H$, Naves T, Vincent F, Magnaudeix A, Durand K, Bertin F, et al. Sortilin limits EGFR signaling by promoting its internalization in lung cancer. Nat Commun. 2017; 8: 1182.

16. Pietila M, Sahgal P, Peuhu E, Jantti NZ, Paatero I, Narva E, et al. SORLA regulates endosomal trafficking and oncogenic fitness of HER2. Nat Commun. 2019; 10: 2340.

17. Al-Akhrass H, Conway JRW, Poulsen ASA, Paatero I, Kaivola J, Padzik A, et al. A feed-forward loop between SorLA and HER3 determines heregulin response and neratinib resistance. Oncogene. 2021; 40: 1300-17.

18. Delivanoglou N, Boziki M, Theotokis P, Kesidou E, Touloumi O, Dafi N, et al. Spatio-temporal expression profile of NGF and the two-receptor system, TrkA and p75NTR, in experimental autoimmune encephalomyelitis. J Neuroinflammation. 2020; 17: 41.

19. Tong B, Pantazopoulou V, Johansson E, Pietras A. The p75 neurotrophin receptor enhances HIFdependent signaling in glioma. Exp Cell Res. 2018; 371: 122-9.

20. Wang TC, Luo SJ, Lin CL, Chang PJ, Chen MF. Modulation of p75 neurotrophin receptor under hypoxic conditions induces migration and invasion of C6 glioma cells. Clin Exp Metastasis. 2015; 32: 73-81.

21. Berghoff J, Jaisimha AV, Duggan S, MacSharry J, McCarthy JV. Gamma-secretase-independent role for cadherin-11 in neurotrophin receptor p75 (p75(NTR)) mediated glioblastoma cell migration. Mol Cell Neurosci. 2015; 69: 41-53. 
22. Wang L, Rahn JJ, Lun X, Sun B, Kelly JJ, Weiss S, et al. Gamma-secretase represents a therapeutic target for the treatment of invasive glioma mediated by the $\mathrm{p} 75$ neurotrophin receptor. PLoS Biol. 2008; 6: e289.

23. Ahn BY, Saldanha-Gama RF, Rahn JJ, Hao X, Zhang J, Dang NH, et al. Glioma invasion mediated by the p75 neurotrophin receptor (p75(NTR)/CD271) requires regulated interaction with PDLIM1. Oncogene. 2016; 35: 1411-22.

24. Yan T, Zhang Z, Li D. NGF receptors and PI3K/AKT pathway involved in glucose fluctuation-induced damage to neurons and alpha-lipoic acid treatment. BMC Neurosci. 2020; 21: 38.

25. Pediaditakis I, Kourgiantaki A, Prousis KC, Potamitis C, Xanthopoulos KP, Zervou M, et al. BNN27, a 17-Spiroepoxy Steroid Derivative, Interacts With and Activates p75 Neurotrophin Receptor, Rescuing Cerebellar Granule Neurons from Apoptosis. Front Pharmacol. 2016; 7: 512.

26. Nykjaer A, Lee R, Teng KK, Jansen P, Madsen P, Nielsen MS, et al. Sortilin is essential for proNGFinduced neuronal cell death. Nature. 2004; 427: 843-8.

27. Naslavsky N, Caplan S. The enigmatic endosome - sorting the ins and outs of endocytic trafficking. J Cell Sci. 2018; 131.

28. Wilson $\mathrm{CM}$, Naves $\mathrm{T}$, Al Akhrass $\mathrm{H}$, Vincent $\mathrm{F}$, Melloni B, Bonnaud $\mathrm{F}$, et al. A new role under sortilin's belt in cancer. Commun Integr Biol. 2016; 9: e1130192.

29. Uchiyama K, Tomita M, Yano M, Chida J, Hara H, Das NR, et al. Prions amplify through degradation of the VPS10P sorting receptor sortilin. PLoS Pathog. 2017; 13: e1006470.

30. Tanimoto R, Palladino C, Xu SQ, Buraschi S, Neill T, Gomella LG, et al. The perlecan-interacting growth factor progranulin regulates ubiquitination, sorting, and lysosomal degradation of sortilin. Matrix Biol. 2017; 64: 27-39.

31. Canuel M, Lefrancois S, Zeng J, Morales CR. AP-1 and retromer play opposite roles in the trafficking of sortilin between the Golgi apparatus and the lysosomes. Biochem Biophys Res Commun. 2008; 366: 724-30.

32. Ni X, Morales CR. The lysosomal trafficking of acid sphingomyelinase is mediated by sortilin and mannose 6-phosphate receptor. Traffic. 2006; 7: 889-902.

33. Okon IS, Coughlan KA, Zhang C, Moriasi C, Ding Y, Song P, et al. Protein kinase LKB1 promotes RAB7mediated neuropilin-1 degradation to inhibit angiogenesis. J Clin Invest. 2014; 124: 4590-602.

\section{Figures}


A

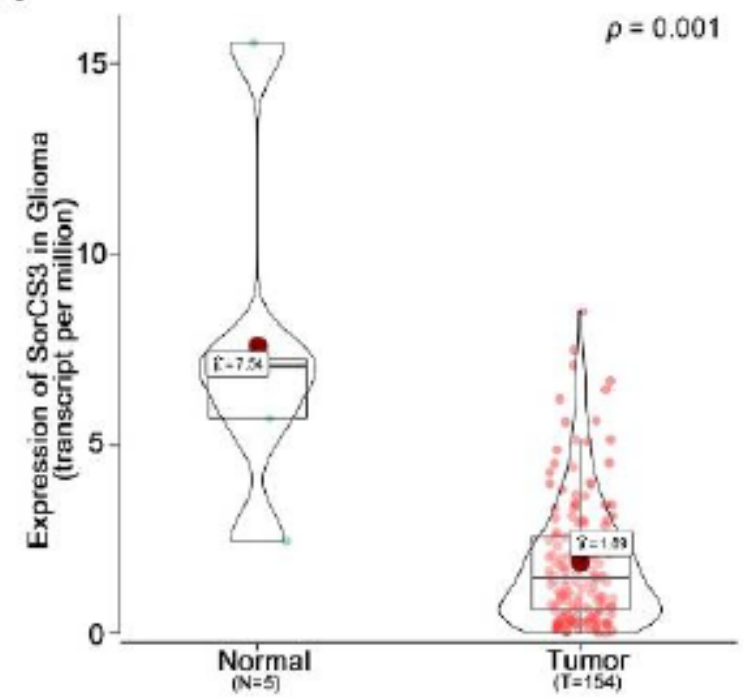

C

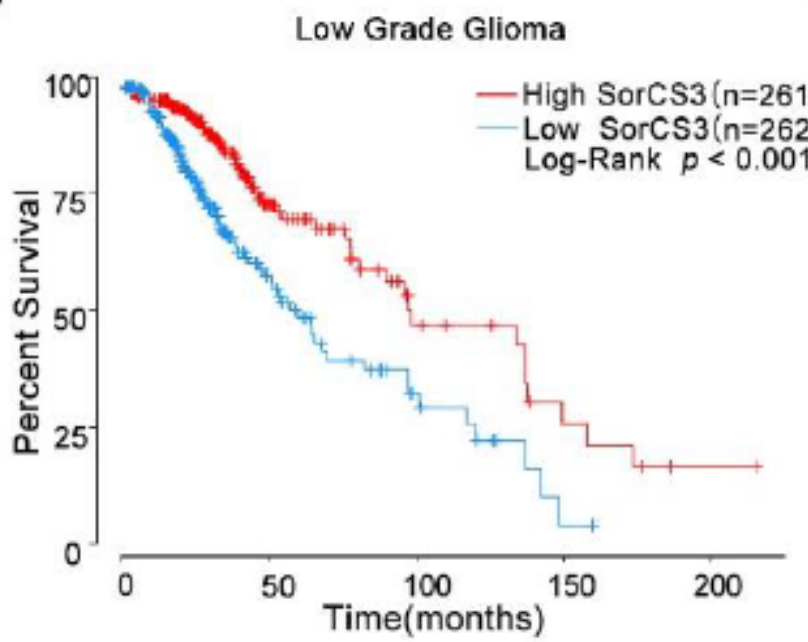

B

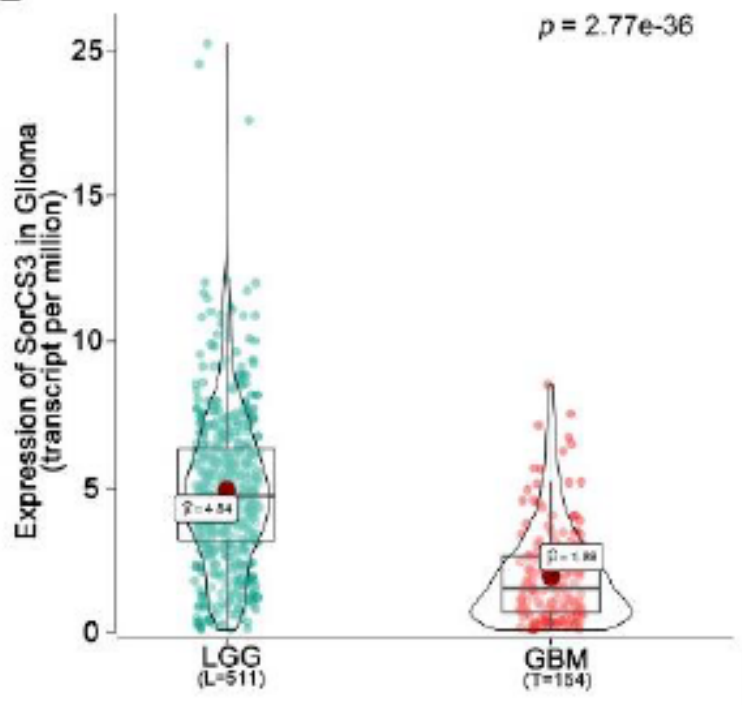

D

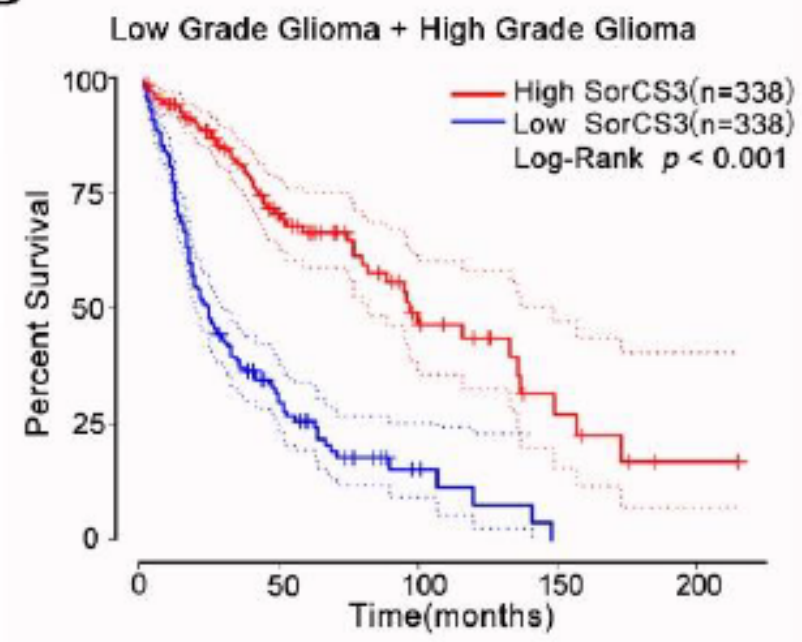

\section{Figure 1}

Overview of SorCS3 expression in TCGA glioma database. (A) SorCS3 expression level in glioma tissues and adjacent normal tissues in the glioma datasets from TCGA. (B) SorCS3 expression level in low grade glioma tissues and GBM in the glioma datasets from TCGA. (C) Kaplan-Meier survival curves of glioma from TCGA with different SorCS3 expression levels $₫ n=523$; High SorCS3, $n=261$; Low SorCS3, $n=262$; $p<0.001 \rrbracket$. (D) Kaplan-Meier Survival curves of different SorCS3 expression levels for Low and High

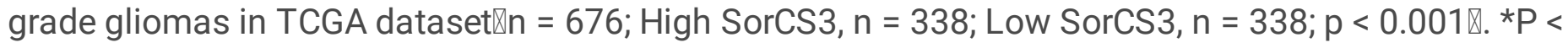
$0.05 ; * \star P<0.01 ; * \star * P<0.001$. 
A

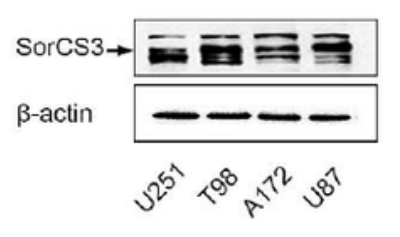

B

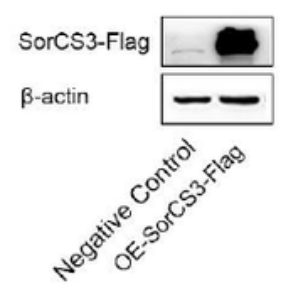

E
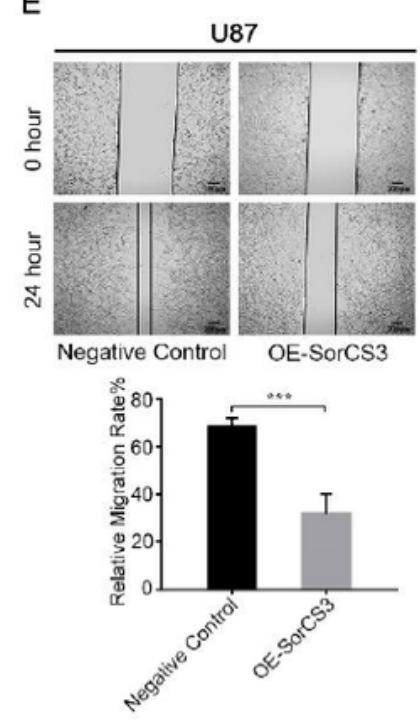

H

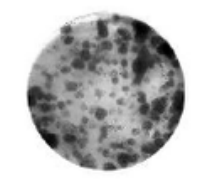

Negative Control
C
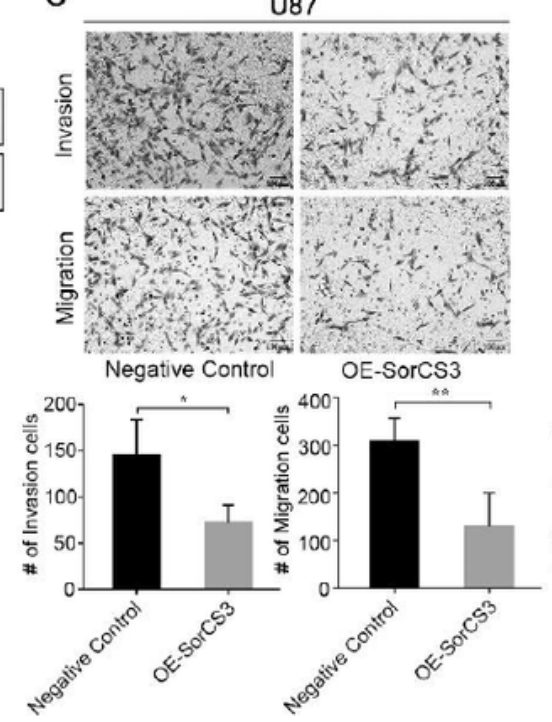

D
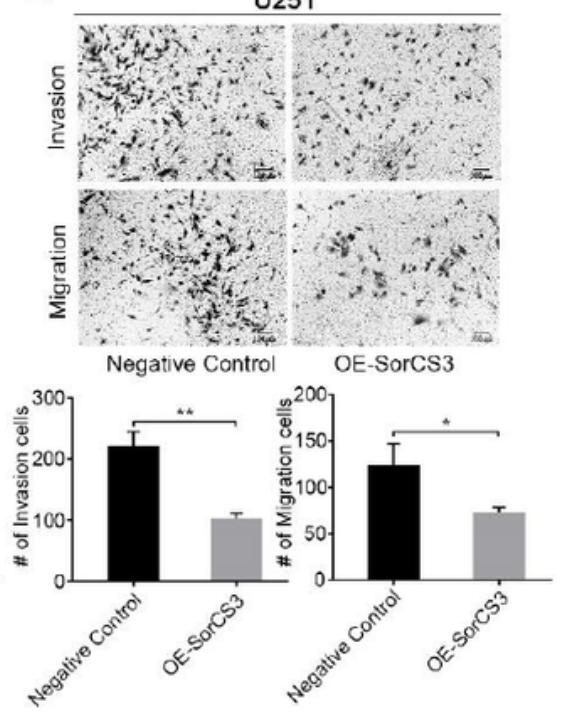

G
F
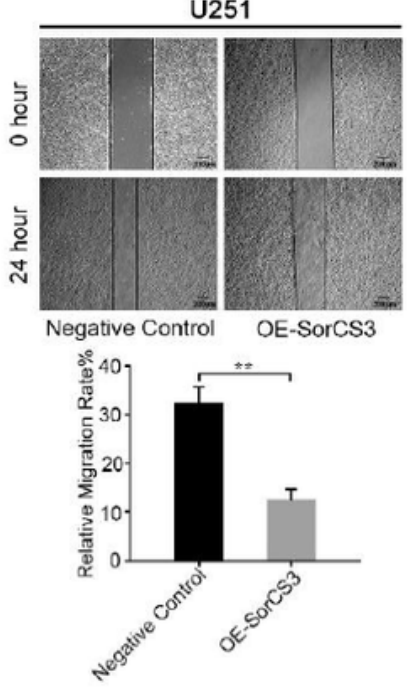
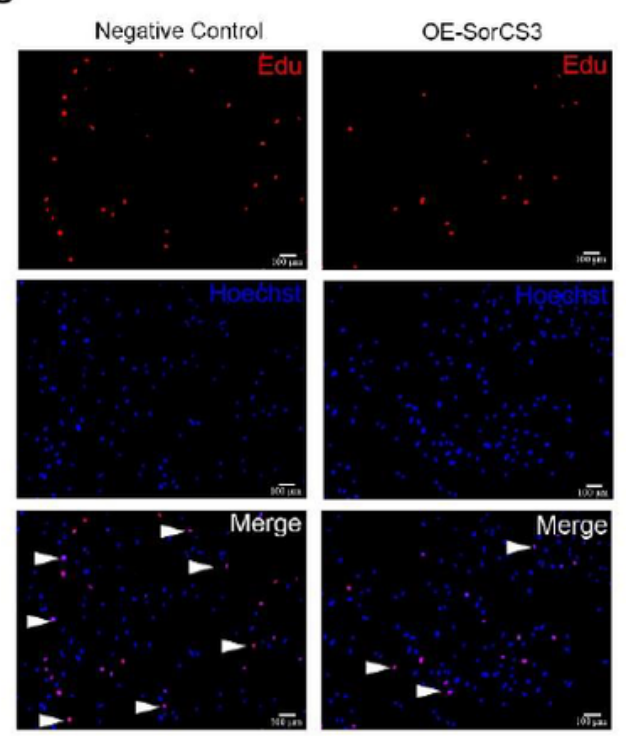

I
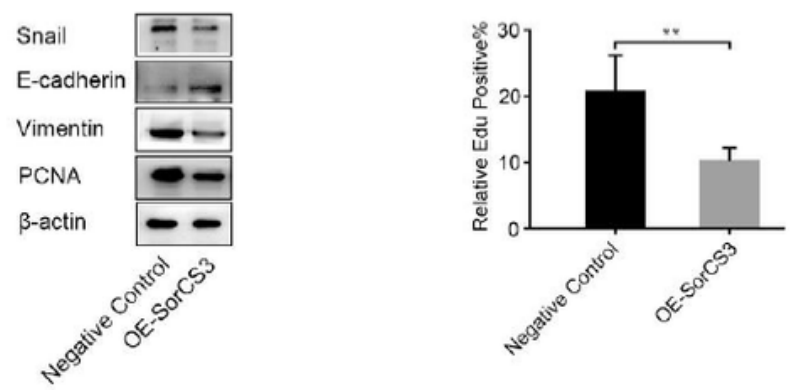

\section{Figure 2}

Overexpression of SorCS3 inhibits the migration, invasion, and proliferation of the glioma cells in vitro. (A) SorCS3 protein levels in glioma cell lines using $\beta$-actin as an endogenous control. (B) SorCS3-Flag expression levels in U251 cells transfected with SorCS3-Flag plasmids were assessed by Western blot. (CD) Transwell assays used to determine the influence of SorCS3 overexpression on the migratory and invasive abilities of U251 and U87 cells. (E-F) Effect of SorCS3 overexpression on wound healing of U251 
and U87 cells. (G) Cell proliferation was determined by EdU staining and EdU incorporation was calculated as EdU+ cells/total cells, quantified by ImageJ. Red was stained for proliferation (Edu+), blue was stained for nucleus. $(\mathrm{H})$ Colony formation assay for assessing the cell proliferation of overexpression SorCS3 in u251 cells. (I) Western blot analysis of proliferation- and EMT-associated marker after SorCS3 overexpression. Data are shown as mean \pm S.D. including three independent experiments. ${ }^{*} \mathrm{P}<0.05 ;{ }^{* *} \mathrm{P}<0.01 ; * * * \mathrm{P}<0.001$.

A
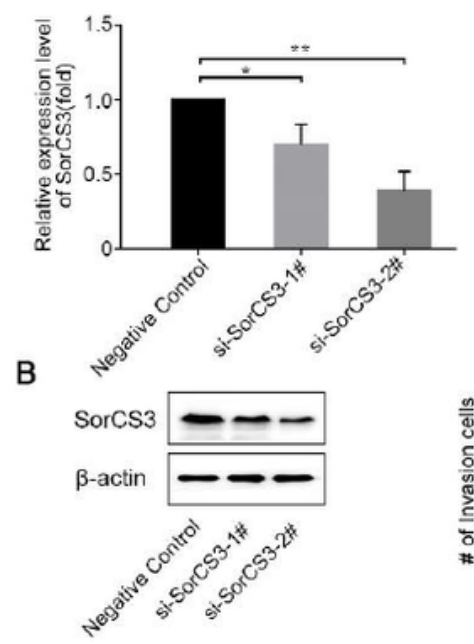

E
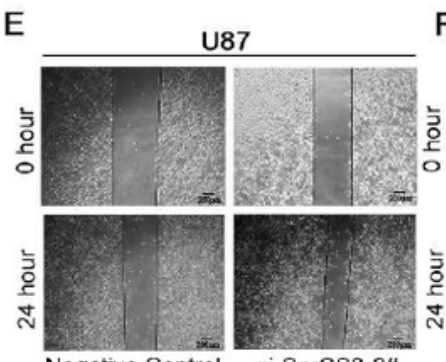

Negative Control si-SorCS3-2\#

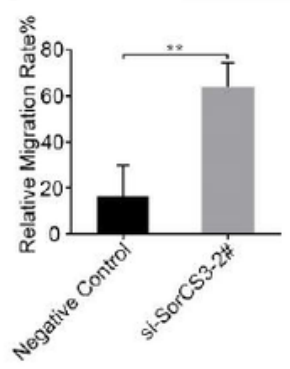

C

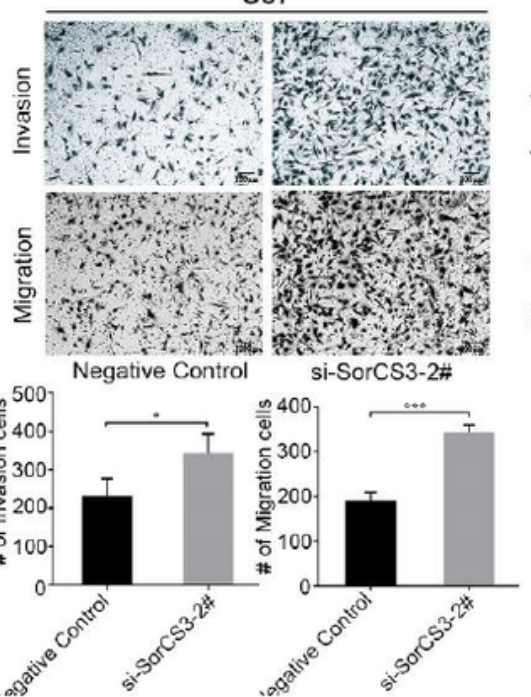

D
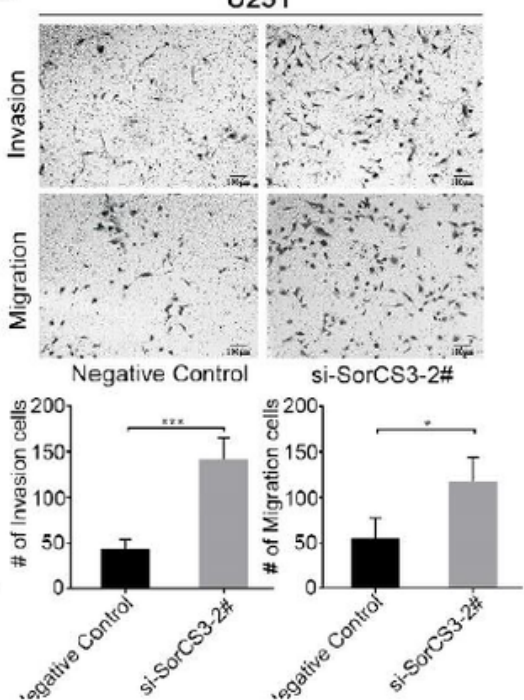

G
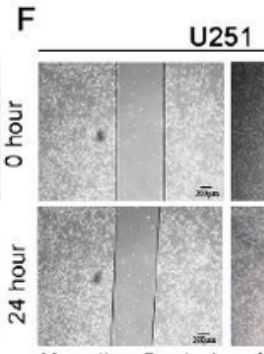

Negative Control

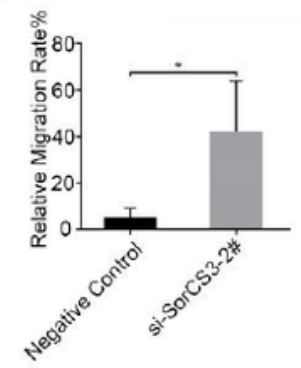

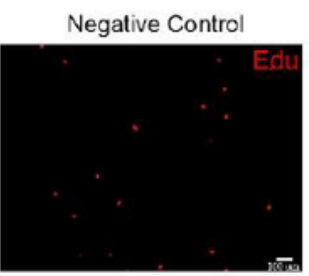
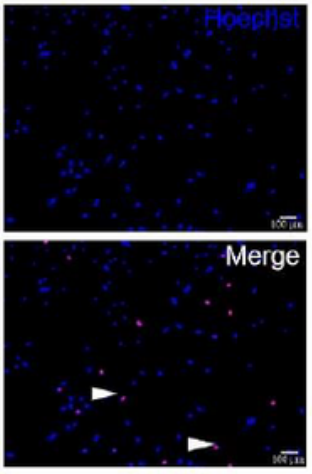

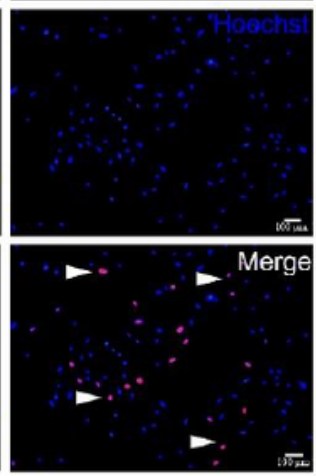

si-SorCs3-2\#

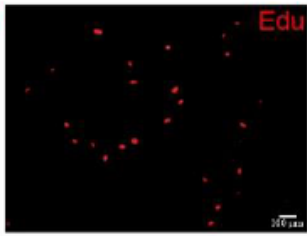

$\mathrm{H}$

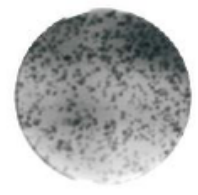

Negative Control

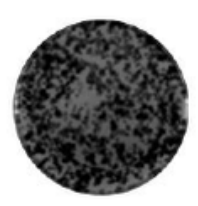

si-SorCs3-2\#
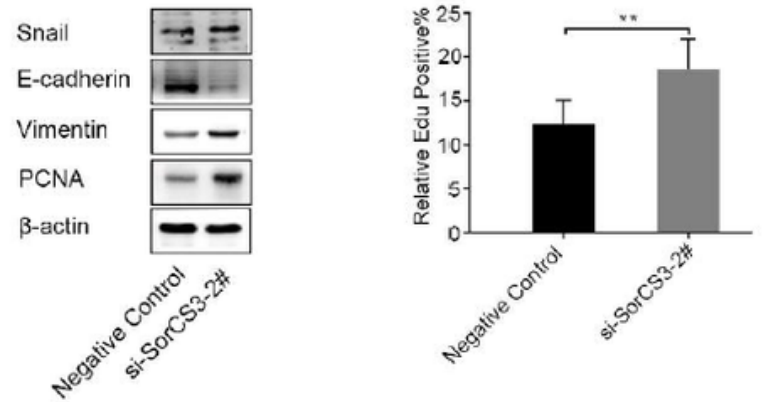

I

Figure 3 
Knockdown of SorCS3 promotes the proliferation, migration and invasion of glioma cells in vitro. (A-B) After siRNA-SorCS3-2\# transfection, the expression levels of SorCS3 were examined through real-time PCR and Western blotting, using $\beta$-actin as an endogenous control. (C-D) Transwell assays used to determine the influence of transfection with SorCS3 siRNA-2\# on the migratory and invasive abilities of U251 and U87 cells. (E-F) Effect of SorCS3 knockdown on wound healing of U251 and U87 cells. (G) Cell proliferation was determined by EdU staining in SorCS3 knockdown condition and EdU incorporation was calculated as EdU+ cells/total cells, quantified by ImageJ. Red was stained for proliferation (Edu+), blue was stained for nucleus. $(\mathrm{H})$ Colony formation assay for assessing the cell proliferation of knockdown SorCS3 in u251 cells. (I) Western blot analysis of proliferation- and EMT-associated marker after transfection with SorCS3 siRNA. Data are shown as mean \pm S.D. including three independent experiments. ${ }^{*} \mathrm{P}<0.05 ; * \star \mathrm{P}<0.01 ; * \star \star \mathrm{P}<0.001$. 
A
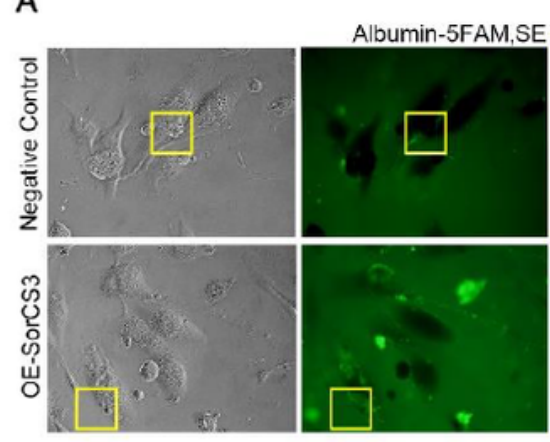

C

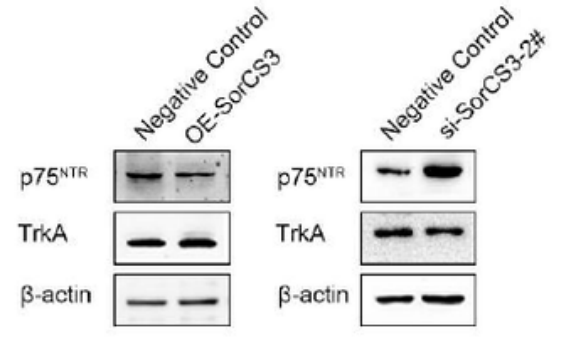

F

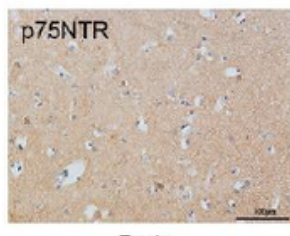

Brain

G

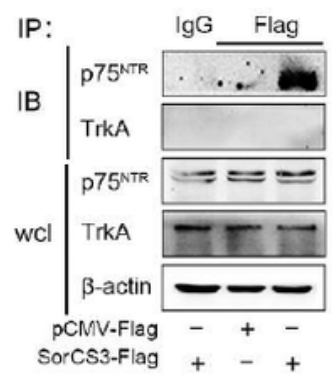

$\mathrm{H}$

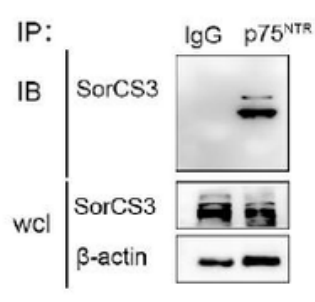

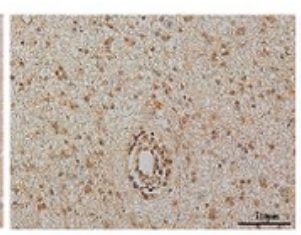

I Grade
B
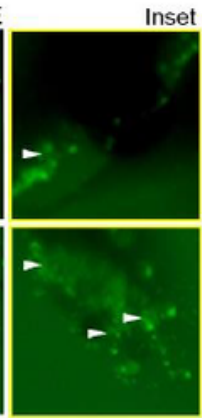

D
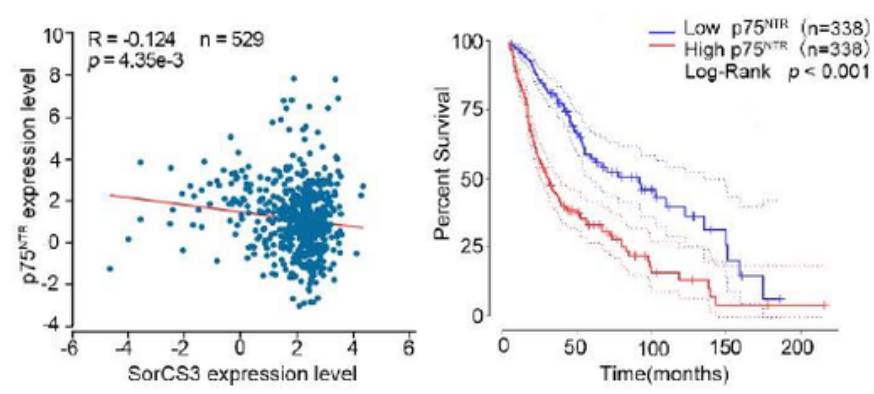

Albumin-5FAM,S
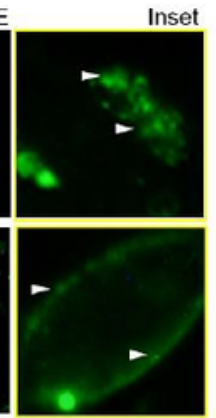

$\square$
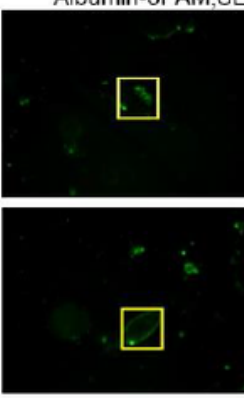

E

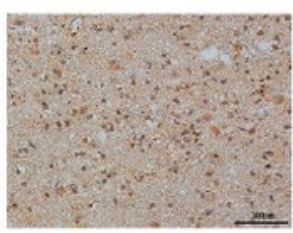

II Grade

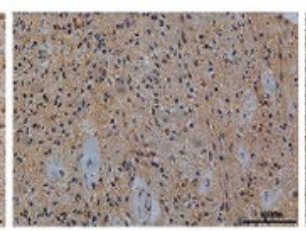

III Grade
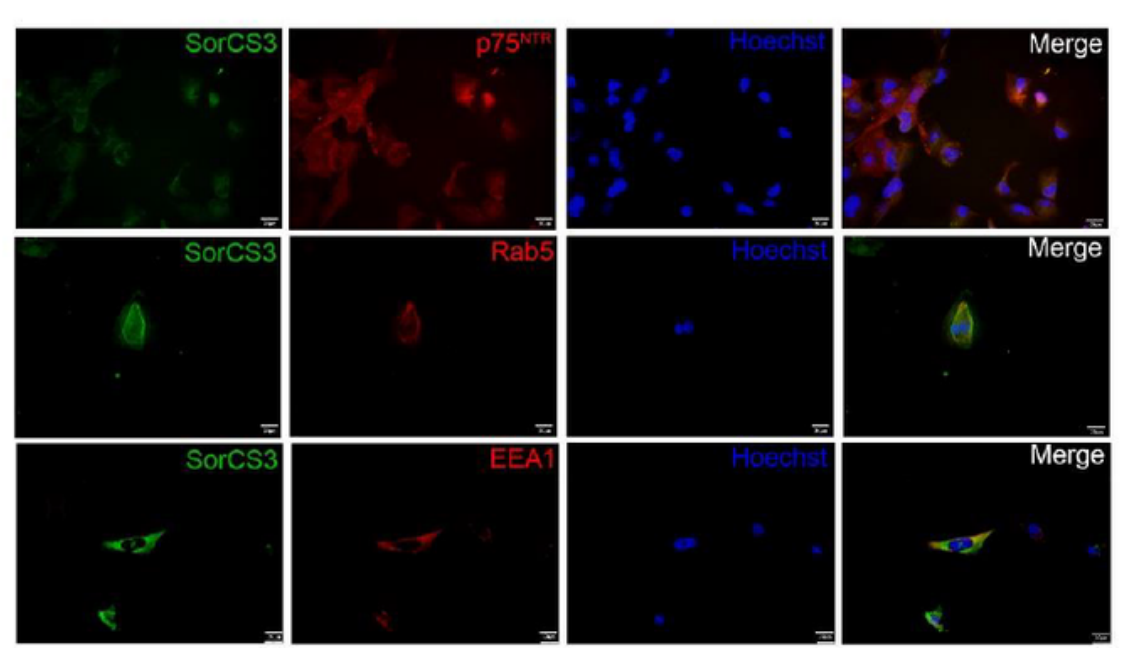

\section{Figure 4}

SorCS3 regulates p75NTR expression and internalization. (A-B) Fluorescence microscopy images of live cells were treated with Albumin-labeled 5-FAM,SE in altered sorcs3 expression condition. (C) Western blot analysis of p75NTR and TrkA levels after altering the expression of SorCS3 in glioma cells. (D) Correlation between SorCS3 andp75NTR mRNA expression levels were analyzed using starBase v2.0. (E) Kaplan-Meier survival curves of glioma from TCGA with different p75NTR expression levels $₫ n$ = 676; High 
SorCS3, $n=338$; Low SorCS3, $n=338$; $\mathrm{P}<0.001 \mathrm{Q}$. (F) P75NTR expression with different WHO grade glioma and normal brain tissues taken from 108 patients was assessed by IHC. Scale bar: $100 \mu \mathrm{m}$. (G-H) Immunoprecipitations were performed using anti-Flag $(\mathrm{G})$ or p75NTR $(\mathrm{H})$ antibody, and the immunocomplexes were immunoblotted (IB) using anti-SorCS3囚p75NTR and Trka antibody in transfected SorCS3-Flag plasmid. In parallel, immunoblots for SorCS3, p75NTR and TrkA were performed on wholecell lysates (WCL); the isotypic lane Immunoglobulin G (IgG) represents the IP control. (I) U87 cells were transfected with SorCS3-Flag plasmid, and then immunolabeled for SorCS3 and markers of the early endosome (Rab5ロEEA1) and the p75NTR. Scale bar, $5 \mu \mathrm{m}$. 
A

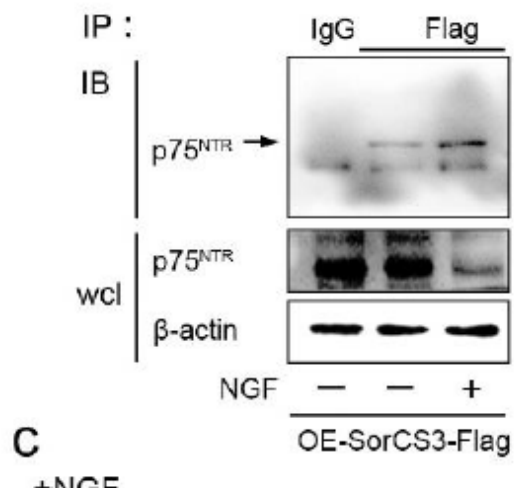

\section{B}

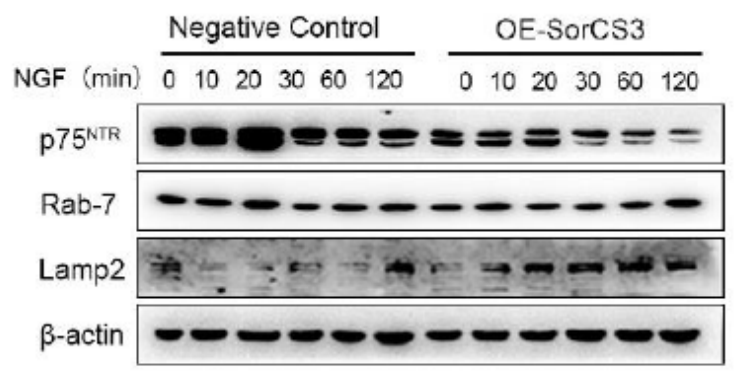

+ NGF
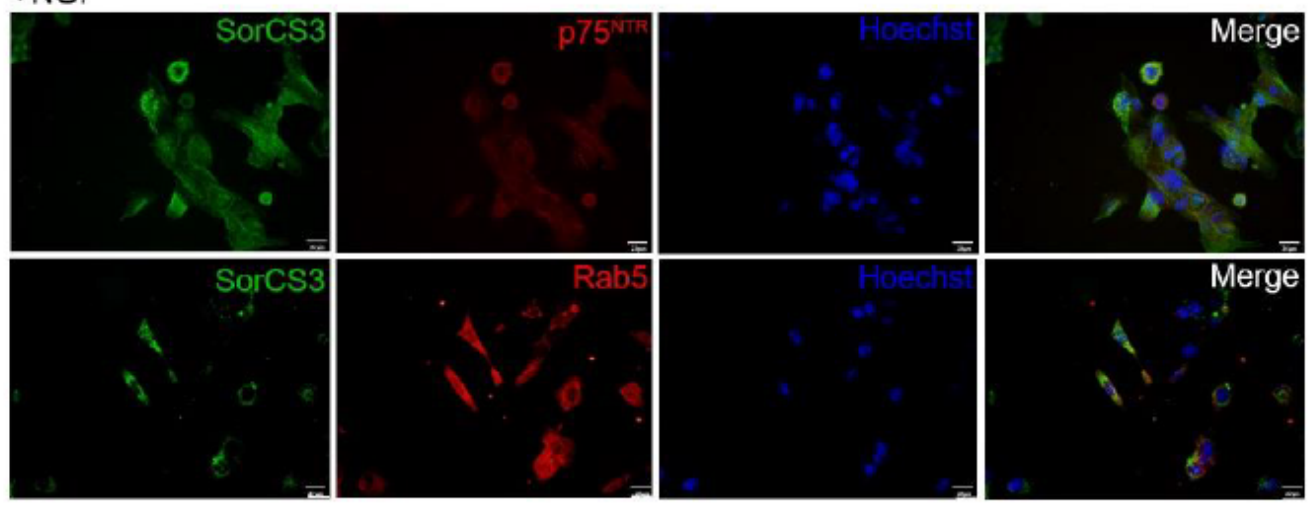

D
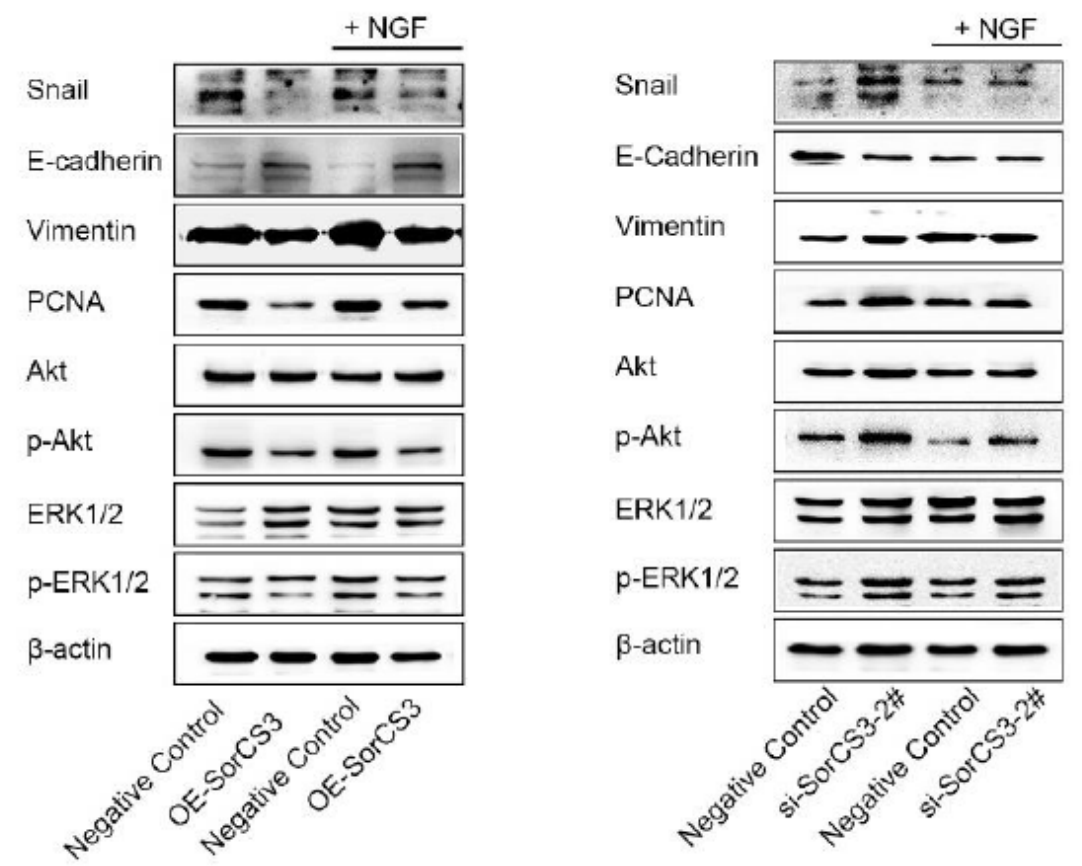

\section{Figure 5}

NGF promotes the SorCS3 and p75NTR interaction. (A) U87 cells grown in complete cell culture media were transfected SorCS3-Flag plasmid, and then stimulated or not with NGF (100 ng/mL) for $60 \mathrm{~min}$. Immunoprecipitations (IP) were performed using anti-Flag antibody, and the immunocomplexes were immunoblotted (IB) using anti-p75NTR antibody. In parallel, immunoblots for p75NTR were performed on whole-cell lysates (WCL); the isotypic lane Immunoglobulin G (IgG) represents the IP control. (B) U87 
overexpression of SorCS3 and control were stimulated with NGF $(100 \mathrm{ng} / \mathrm{mL})$ over a 60 min time course. Cell lysates were analyzed by western blotting for components of the canonical internalization signaling pathway using the indicated antibodies. (C) U87 cells were stimulated with NGF (100 ng/mL) for 60 min, and then immunofluorescence for SorCS3-Flag, p75NTR and markers of the early endosome (Rab5). Scale bar, $5 \mu \mathrm{m}$. (D) U87 were transfected with SorCS3-Flag plasmid or siRNA (si-SorCS3-2\#) and the corresponding control, and then stimulated with NGF $(100 \mathrm{ng} / \mathrm{mL})$ for $60 \mathrm{~min}$. Cell lysates were analyzed by western blotting with the indicated antibodies, using $\beta$-actin as an endogenous control.

A

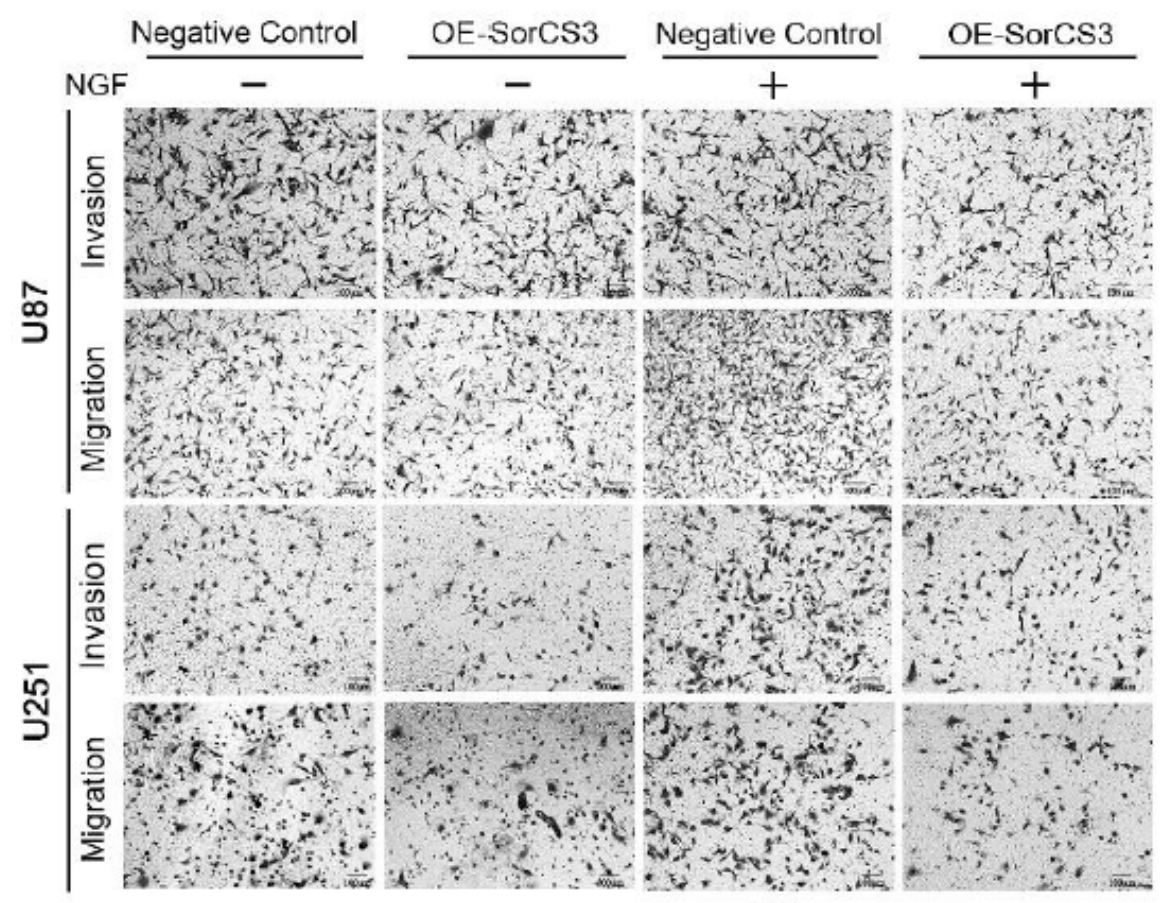

B
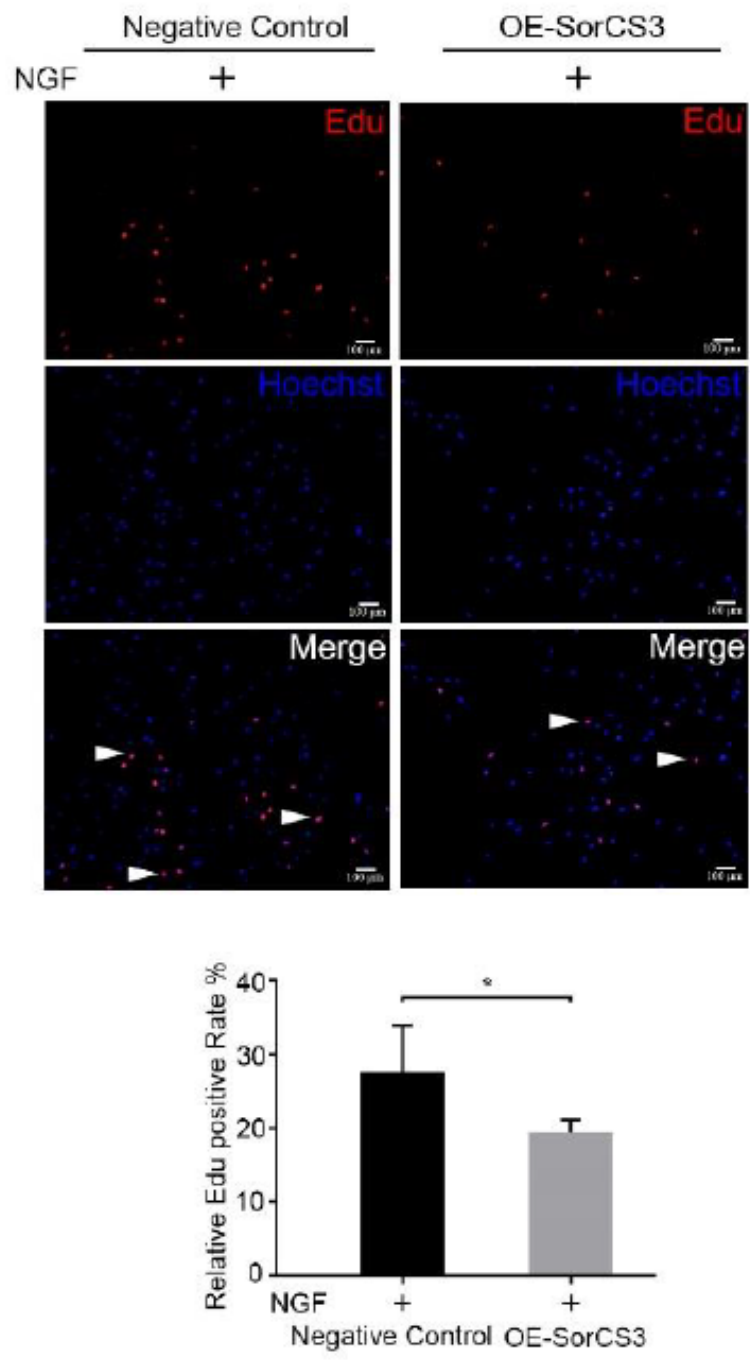
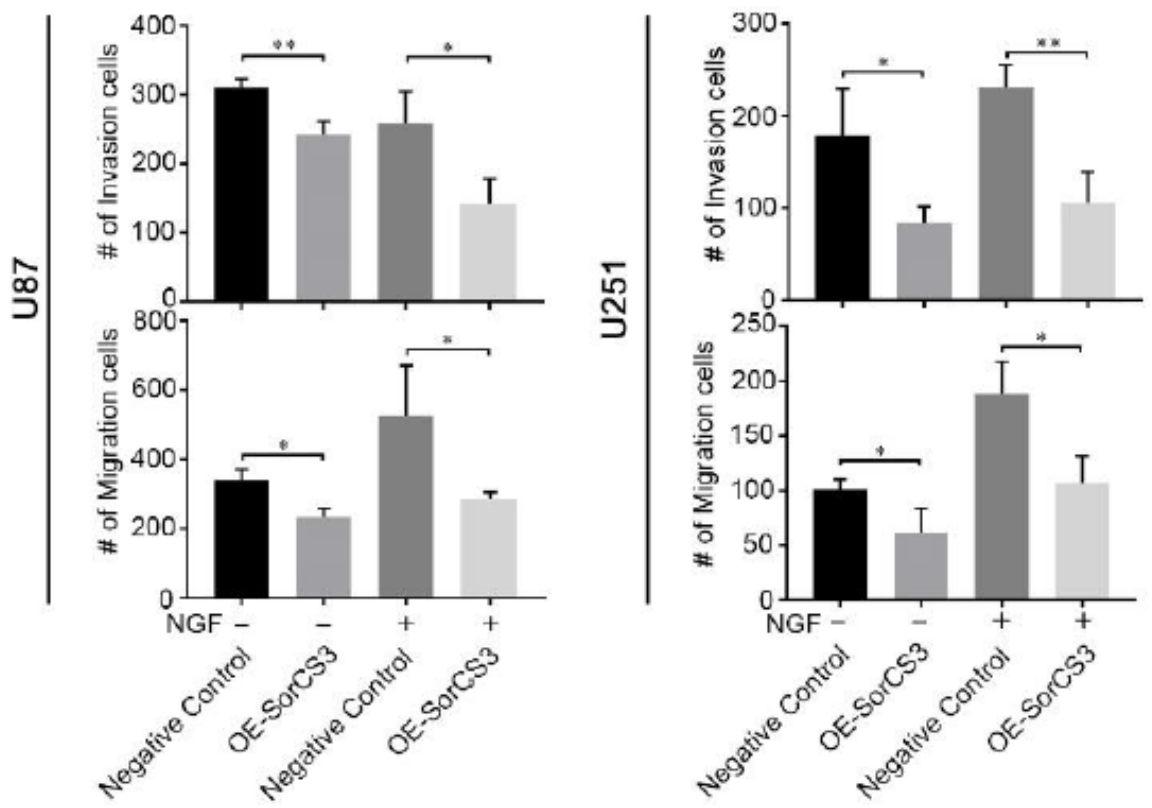

Figure 6 
Stimulation of NGF improves the ability of SorCS3 to inhibit the malignant phenotype of glioma cell lines. (A-B) Transwell assays used to determine the influence of SorCS3 overexpression stimulated or not with NGF (100 ng/mL) for 60 min on the migratory and invasive abilities of U251 and U87 cells. (C) EdU staining to determine the influence of SorCS3 overexpression stimulated or not with NGF $(100 \mathrm{ng} / \mathrm{mL})$ for $60 \mathrm{~min}$, and EdU incorporation was calculated as EdU+ cells/total cells, quantified by ImageJ. Red was stained for proliferation (Edu+), blue was stained for nucleus. Data are shown as mean \pm S.D. including three independent experiments. ${ }^{*} \mathrm{P}<0.05 ;{ }^{*} \mathrm{P}<0.01$.

A

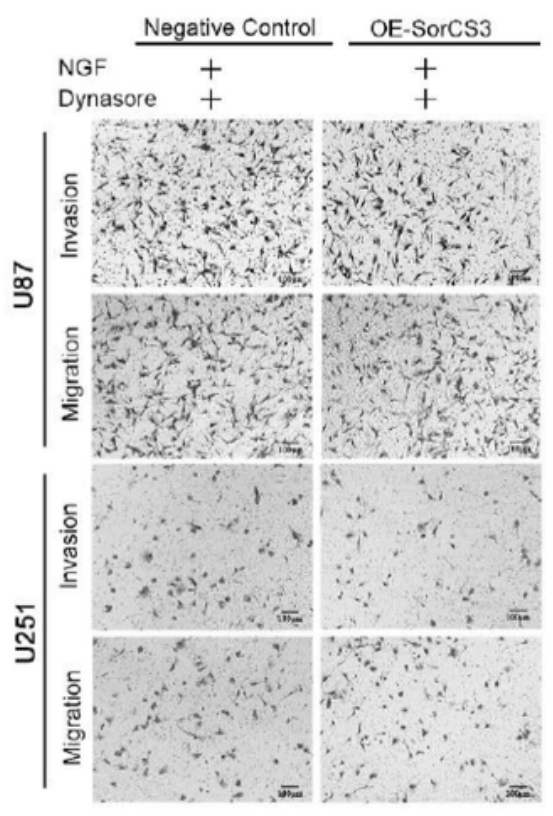

B
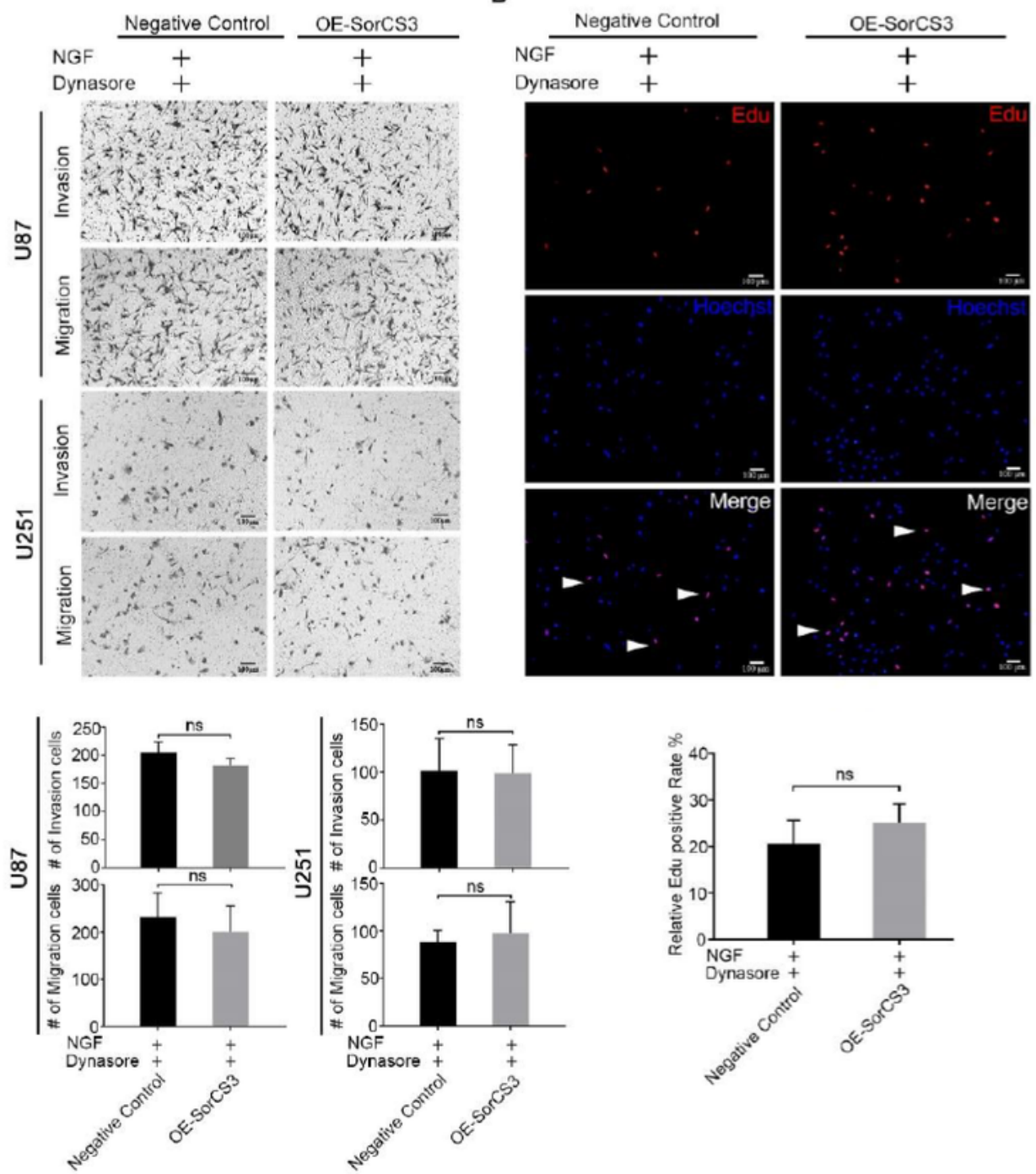

C

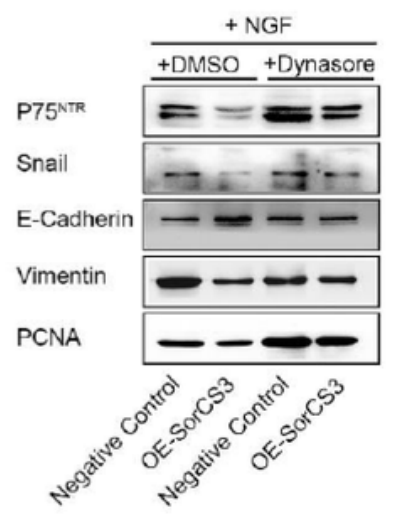

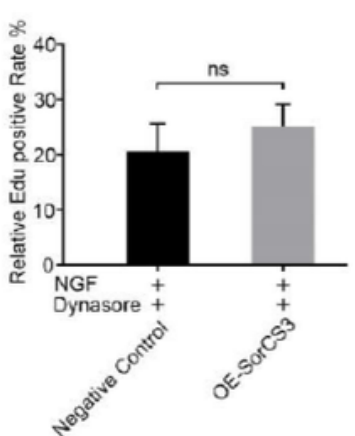

D

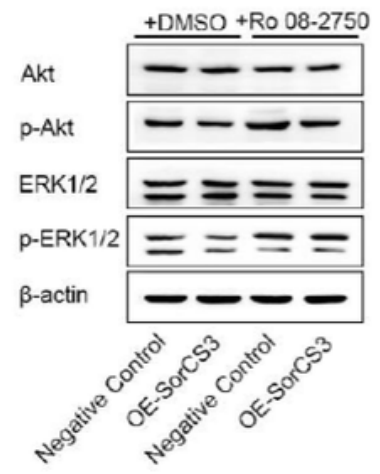




\section{Figure 7}

Inhibition of internalize abolishes the tumor suppressor effects of SorCS3. (A) Transwell assays were used to determine the influence of U87 and U251 cells pretreated with the cell-permeable dynamin inhibitor Dynasore $(40 \mu \mathrm{M})$ for 2 hours and then stimulated with NGF $(100 \mathrm{ng} / \mathrm{mL})$ for $60 \mathrm{~min}$ in overexpression of SorCS3 condition. (B) EdU staining to determine the influence of SorCS3 overexpression pretreated with the cell-permeable dynamin inhibitor Dynasore $(40 \mu \mathrm{M})$ for 2 hours and then stimulated with NGF $(100 \mathrm{ng} / \mathrm{mL})$ for $60 \mathrm{~min}$, and EdU incorporation was calculated as EdU+ cells/total cells, quantified by ImageJ. Red was stained for proliferation (Edu+), blue was stained for nucleus. (C) U87 were pretreated with the cell-permeable dynamin inhibitor Dynasore $(40 \mu \mathrm{M})$ for 2 hours and then stimulated with NGF $(100 \mathrm{ng} / \mathrm{mL})$ for 60 min in overexpression of SorCS3 condition. Cell lysates were analyzed by western blotting with the indicated antibodies, using $\beta$-actin as an endogenous control. (D) U87 were pretreated with the Ro 08-2750 ( $1 \mu \mathrm{M}$, Ro 08-2750 is a reversible NGF inhibitor which inhibits NGF binding to p75NTR selectively) for 8 hours and then stimulated with NGF $(100 \mathrm{ng} / \mathrm{mL})$ for 60 min in overexpression of SorCS3 condition. Cell lysates were analyzed by western blotting with the indicated antibodies, using $\beta$-actin as an endogenous control. Data are shown as mean \pm S.D. including three independent experiments. ns, no significance.

A

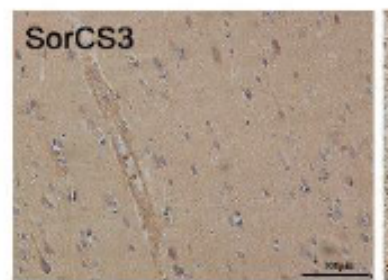

Brain

B

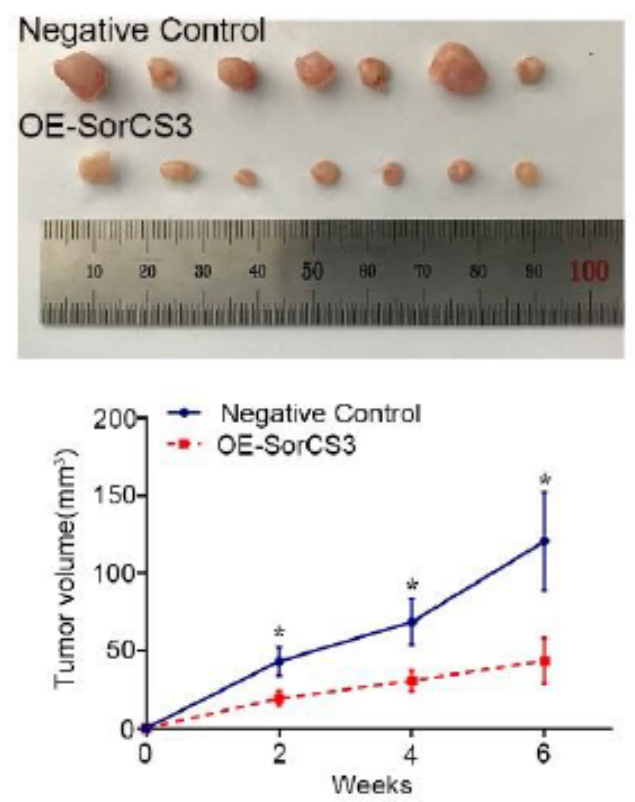

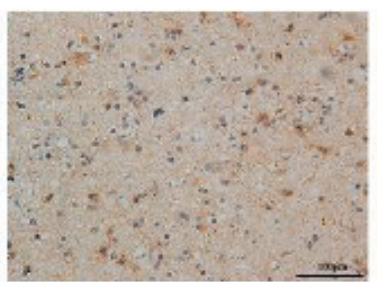

II Grade

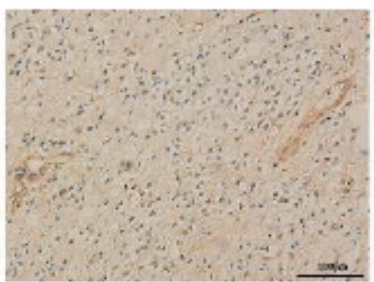

III Grade

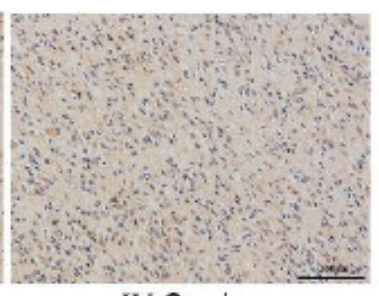

IV Grade

C

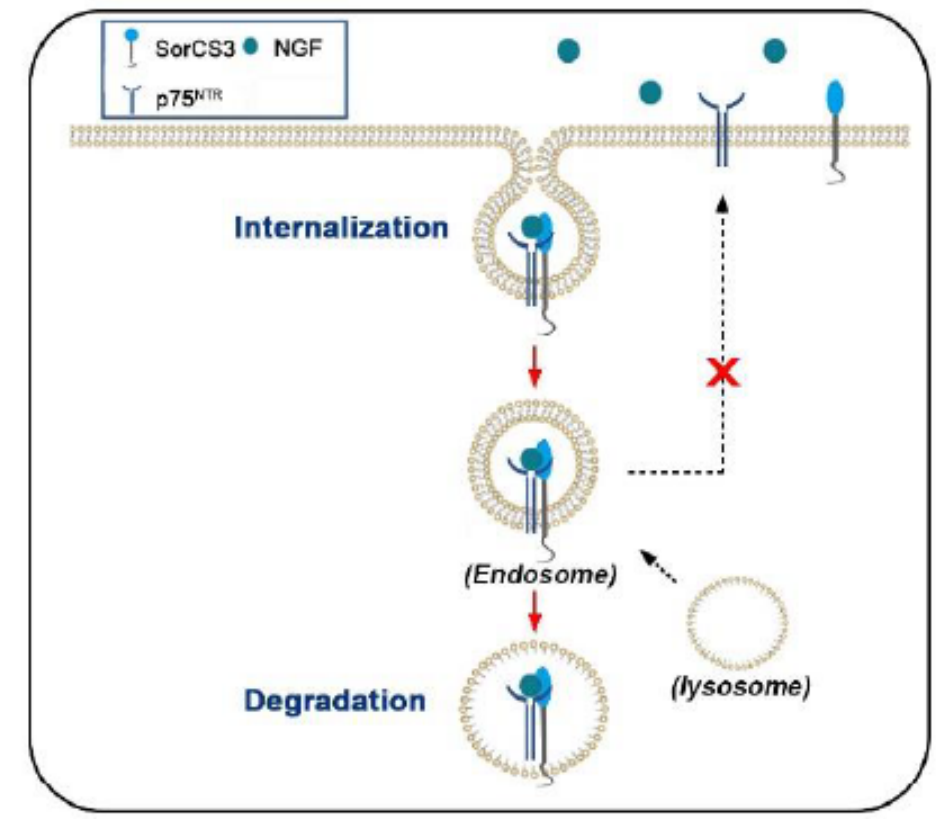

Figure 8 
SorCS3 expression decreased with the tumor aggressiveness. (A) SorCS3 expression with different WHO grade glioma and normal brain tissues taken from 108 patients was assessed by IHC. Scale bar: $100 \mu \mathrm{m}$. (B) Image of tumors in nude mice bearing U87 cells treated with overexpression of SorCS3 and control. (C) Tumor volume (C) and tumor weight (D) in (B) were measured $(n=7)$. (E) SorCS3 exerts tumor suppressor effect by enhance p75NTR internalization and transport to lysosome degradation in glioma. Data are shown as mean \pm S.D. including three independent experiments. ${ }^{*} P<0.05$.

\section{Supplementary Files}

This is a list of supplementary files associated with this preprint. Click to download.

- Additionalfile.pdf 